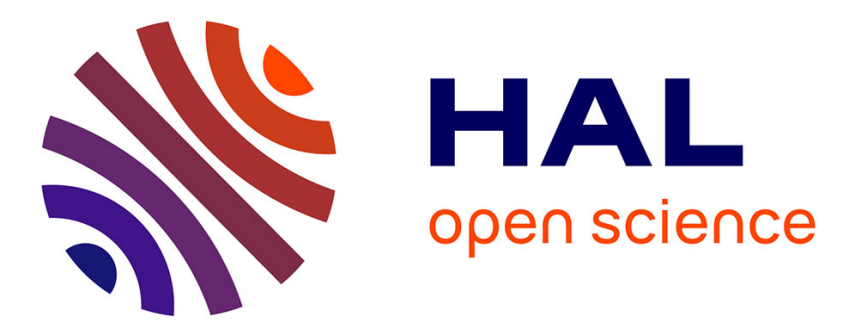

\title{
The descriptive complexity of connectedness in Polish spaces
}

Gabriel Debs, Jean Saint Raymond

\section{To cite this version:}

Gabriel Debs, Jean Saint Raymond. The descriptive complexity of connectedness in Polish spaces. Fundamenta Mathematicae, 2020, 249 (3), pp.261-286. 10.4064/fm754-7-2019 . hal-02955782

\section{HAL Id: hal-02955782 \\ https://hal.sorbonne-universite.fr/hal-02955782}

Submitted on 2 Oct 2020

HAL is a multi-disciplinary open access archive for the deposit and dissemination of scientific research documents, whether they are published or not. The documents may come from teaching and research institutions in France or abroad, or from public or private research centers.
L'archive ouverte pluridisciplinaire HAL, est destinée au dépôt et à la diffusion de documents scientifiques de niveau recherche, publiés ou non, émanant des établissements d'enseignement et de recherche français ou étrangers, des laboratoires publics ou privés. 


\title{
The descriptive complexity of connectedness in Polish spaces
}

\author{
Gabriel Debs \\ Sorbonne Université, Université Paris Diderot, CNRS, \\ Institut de Mathématiques de Jussieu-Paris Rive Gauche, IMJ-PRG, \\ 4 place Jussieu, \\ F-75252, Paris, France
}

and Université Le Havre Normandie, Institut Universitaire de Technologie, Rue Boris Vian, BP 4006, 76610 Le Havre, France

Email: gabriel.debs@imj-prg.fr

Jean Saint Raymond

Sorbonne Université, Université Paris Diderot, CNRS,

Institut de Mathématiques de Jussieu-Paris Rive Gauche, IMJ-PRG,

4 place Jussieu, F-75252, Paris, France

Email: jean.saint-raymond@imj-prg.fr

\begin{abstract}
We investigate the descriptive complexity of connectedness (pathwise connectedness, local connectedness) of Polish spaces, and prove that even in the frame of finite dimensional euclidean spaces this complexity can be as high as possible, and much beyond the first projective classes $\boldsymbol{\Sigma}_{1}^{1}$ and $\boldsymbol{\Pi}_{1}^{1}$. In particular we prove that several of these notions are $\boldsymbol{\Pi}_{2}^{1}$-complete.
\end{abstract}

\section{Introduction}

This work is a continuation of [2] where was investigated the maximal possible complexity of the set of all zero-dimensional closed subsets of a given Polish space. In the present work we study the analogous question for connectedness which is a kind of strong negation of zero-dimension. In fact we shall investigate this question for several variations of the notion of connectedness. More precisely we shall compute the maximal possible complexity, for a given Polish space $X$, of the following sets :

\footnotetext{
2010 Mathematics Subject Classification: Primary 03E15, 28A05, 54D05; Secondary 54H05 Key words and phrases:Connectedness, local connectedness, Effros Borel strucure, projective sets.
} 
$\mathcal{C}(X)$ : the set of all closed connected subsets of $X$,

$\check{\mathcal{C}}(X)$ : the set of all closed subsets of $X$ whose complement is connected,

$\mathcal{C}_{\text {path }}(X):$ the set of all closed pathwise connected subsets of $X$,

$\mathcal{C}_{\text {loc }}(X)$ : the set of all closed locally connected subsets of $X$.

It turns out that this complexity depends on whether the ground space $X$ is compact or merely Polish, as shown by the following table which gives upper bounds for the complexity of these sets:

\begin{tabular}{|c|c|c|c|c|}
\hline & $\mathcal{C}(X)$ & $\check{\mathcal{C}}(X)$ & $\mathcal{C}_{\text {path }}(X)$ & $\mathcal{C}_{\text {loc }}(X)$ \\
\hline$X$ compact & $\Pi_{1}^{0}$ & $\Pi_{1}^{1}$ & $\Pi_{2}^{1}$ & $\Pi_{3}^{0}$ \\
\hline$X$ Polish & $\Pi_{2}^{1}$ & $\Pi_{2}^{1}$ & $\Pi_{2}^{1}$ & $\check{\mathcal{A}}\left(\mathbf{\Pi}_{1}^{1}\right)$ \\
\hline
\end{tabular}

Note that in the compact case the complexity is computed in the compact space $\mathcal{K}(X)$ of all compact subsets of $X$, while in the Polish case the complexity is relatively to the canonical Borel structure of the space $\mathcal{F}(X)$ of all closed subsets of $X$ (see Section 2). These bounds are obtained by a straightforward expansion of definitions, except the bound $\check{\mathcal{A}}\left(\boldsymbol{\Pi}_{1}^{1}\right)$ (see Section 3 for the notation) which relies on some specific analysis and properties of Polish locally connected spaces. As we shall see all these bounds are optimal. More precisely we shall prove:

a) There exists a Polish space $X \subset \mathbb{R}^{3}$ with $\mathcal{C}(X) \boldsymbol{\Pi}_{2}^{1}$-complete.

b) There exists a Polish space $X \subset \mathbb{R}^{2}$ with $\breve{\mathcal{C}}(X) \Pi_{2}^{1}$-complete.

c) There exists a compact space $X \subset \mathbb{R}^{3}$ with $\check{\mathcal{C}}(X) \Pi_{1}^{1}$-complete.

d) There exists a Polish space $X \subset \mathbb{R}^{3}$ with $\mathcal{C}_{\text {loc }}(X) \check{\mathcal{A}}\left(\mathbf{\Pi}_{1}^{1}\right)$-complete.

We point out that by a result of Ajtaj and Becker (see [5], Theorem 37.11) the set $\mathcal{C}_{\text {path }}\left(\mathbb{R}^{n}\right)$ is $\boldsymbol{\Pi}_{2}^{1}$-complete for all $n \geq 3$, and following the methods of the present work we shall give in Section 5 an alternative proof of this result for $n \geq 4$.

Observe that since any Polish space can be embedded as a closed subset of the Polish space $\mathbb{R}^{\omega}$ it follows from the previous results that the sets $\mathcal{C}\left(\mathbb{R}^{\omega}\right)$ and $\mathcal{C}_{\text {path }}\left(\mathbb{R}^{\omega}\right)$ are $\boldsymbol{\Pi}_{2}^{1}$-complete, while the set $\mathcal{C}_{\text {loc }}\left(\mathbb{R}^{\omega}\right)$ is $\check{\mathcal{A}}\left(\boldsymbol{\Pi}_{1}^{1}\right)$-complete. Note however that as we shall see (Proposition 7.5) the set $\check{\mathcal{C}}\left(\mathbb{R}^{\omega}\right)$ is actually Borel.

To finish we list next some open questions.

1) There is a priori no topological reason which prevents a 2-dimensional version of the previous results by imposing that $X \subset \mathbb{R}^{2}$. However, aside in 
case b), the constructions used in the paper, which necessitate some geometrical freedom in the surrounding euclidean space, are not easily adaptable to provide such a requirement.

2) These results give the maximal complexity of the considered sets. They do not describe the whole spectrum of possible complexity of these sets for an arbitrary Polish space $X$.

3) One can try to release the restriction on the space $X$ of being Polish. In particular one can consider the same questions for an analytic space $X$. We recall that in this case the canonical Borel structure on $\mathcal{F}(X)$ is isomorphic to the Borel structure of a $\Sigma_{1}^{1}$ subset of $2^{\omega}$, and considering descriptive complexity of subsets of $\mathcal{F}(X)$ is meaningful. Then resuming the same computation than in the Polish case one can check that for an analytic space $X$ the sets $\mathcal{C}(X)$ and $\check{\mathcal{C}}(X)$ are still $\Pi_{2}^{1}$, while the natural complexity upper bound of the sets $\mathcal{C}_{\text {path }}(X)$ and $\mathcal{C}_{\text {loc }}(X)$ jumps up to $\boldsymbol{\Pi}_{4}^{1}$; and it is not clear whether in the two latter cases this bound is optimal.

4) Finally note that one can investigate similarly the descriptive complexity of various topological notions other than connectedness. We mention in particular the case of the set $\mathcal{F}_{0}(X)$ of all closed zero-dimensional subsets of a Polish space $X$. It is not difficult to check that $\mathcal{F}_{0}(X)$ is a $\boldsymbol{\Sigma}_{2}^{1}$ set, and it follows from the results of [2] that the maximal complexity of this set is at least $\partial \boldsymbol{\Sigma}_{2}^{0}$ (see [8] or [2] for the definition of this class of $\boldsymbol{\Delta}_{2}^{1}$ sets). But the maximal complexity of the set $\mathcal{F}_{0}(X)$ is unknown.

We would like to thank the Referee who pointed to us the result of Ajtaj and Becker mentioned above and for his very careful reading of the paper.

\section{Descriptive Notions and Notations}

Along the paper we work in the frame of Polish spaces and we assume the reader to be familiar with basic notions and results from Descriptive Set Theory in this setting, for example as presented in [6] or [8]. We recall that the notion of complexity of a set $A$ is a priori relative to some specified space $X$ containing $A$. However when the surrounding space $X$ is not specified the complexity is to be understood in the maximal sense, that is the complexity relatively to any Polish space $X$ in which $A$ can be embedded.

We shall mainly consider the classical pointclasses of Borel and projective sets, namely:

$\Sigma_{\xi}^{0}$ : the additive Baire class of rank $\xi$

$\boldsymbol{\Pi}_{\xi}^{0}$ : the multiplicative Baire class of rank $\xi\left(\right.$ so $\left.\boldsymbol{\Pi}_{2}^{0}=\mathbf{G}_{\delta}\right)$ 
$\boldsymbol{\Sigma}_{1}^{1}$ : the class of analytic sets.

$\boldsymbol{\Pi}_{1}^{1}$ : the class of co-analytic sets.

$\Delta_{1}^{1}$ : the class of Borel sets.

$\Sigma_{2}^{1}$ : the class of projections of co-analytic sets, also denoted by $P C A$.

$\Pi_{2}^{1}$ : the class of complements of $P C A$ sets, also denoted by $C P C A$.

$\boldsymbol{\Delta}_{2}^{1}:=\boldsymbol{\Sigma}_{2}^{1} \cap \boldsymbol{\Pi}_{2}^{1}$

Also given any pointclass $\Gamma$ we denote by $\check{\Gamma}$ its dual class, so for any Polish space $X, \check{\Gamma}(X)=\{X \backslash A: A \in \Gamma\}$; and by $\mathcal{A}(\Gamma)$ the class of all sets obtained by operation $\mathcal{A}$ applied to a Souslin scheme of sets from $\Gamma$. It is well known that $\mathcal{A}\left(\boldsymbol{\Sigma}_{1}^{1}\right)=\boldsymbol{\Sigma}_{1}^{1}$ and we shall consider in the sequel the class $\mathcal{A}\left(\boldsymbol{\Pi}_{1}^{1}\right)$ and its dual class $\breve{\mathcal{A}}\left(\boldsymbol{\Pi}_{1}^{1}\right)$ which are both subclasses of the class of $C$-sets, hence of the class $\boldsymbol{\Delta}_{2}^{1}$ (see [1] or [2] for more details).

The basic tool for computing complexity is the notion of reduction. We recall that a mapping $\varphi: X \rightarrow Y$ is said to reduce the set $A \subset X$ to the set $B \subset Y$ if $A=\varphi^{-1}(B)$. If $\Lambda$ is a class of functions, $A \subset X$ is said to be $\Lambda$-reducible to $B \subset Y$ if there exists $\varphi: X \rightarrow Y$ in $\Lambda$ which reduces $A$ to $B$; and when $\Lambda$ is the class of continuous functions we simply say that $A \subset X$ is reducible to $B \subset Y$. Note that in writing $A \subset X$ we mean a pair $(A, X)$ such that $A \subset X$, and similarly for $B \subset Y$; and reducibility is actually a relation between such pairs. However when there is no ambiguity on the surrounding spaces $X$ and $Y$, we shall simply say that the set $A$ is reducible to the set $B$. As a matter of fact in many instances, if we impose on the spaces $X$ and $Y$ to be Polish, the notion of reducibility is absolute in the sense that it does not depend on the specific Polish spaces in which the sets $A$ and $B$ are embedded.

If $\Lambda$ is a class of functions between Polish spaces and $\Gamma$ is a descriptive pointclass of subsets of Polish spaces, and $B$ is an arbitrary subset of the Polish space $Y$ then the set $B$ (or more precisely the pair $B \subset Y$ ) is said to be $\Lambda$ - $\Gamma$-hard if any set $A \subset 2^{\omega}$ in $\Gamma$ is reducible to $B$ by a function $\varphi: 2^{\omega} \rightarrow Y$ in $\Lambda$; and if moreover $B$ is in $\Gamma$ then the set $B$ is said to be $\Lambda$ - $\Gamma$-complete. Again when $\Lambda$ is the class of continuous functions we simply say $\Gamma$ - hard and $\Gamma$-complete.

From the proof of the main result of [4] we have the following (see also [3] for a generalization of this result):

Theorem 2.1. (Kechris [4]) Let $\Gamma$ be a pointclass containing the class $\boldsymbol{\Pi}_{1}^{1}$ and closed under Borel substitutions. If a set $B \subset 2^{\omega}$ is Borel- $\Gamma$-hard then $B$ is $\Gamma$-hard.

Note that since any uncountable Polish space is Borel-isomorphic to $2^{\omega}$, it follows from Theorem 2.1 that for a projective class $\Gamma$, being $\Gamma$-complete for a subset $B$ of some Polish space $Y$ is absolute and does not depend on $Y$. 
In all the sequel we denote by $\mathbb{I}$ and $\mathbb{J}$ the intervals $[0,1]$ and $[-1,1]$ respectively.

\section{$3 \quad$ Topologies and Borel Structures on $\mathcal{F}(X)$}

\subsection{The topological space $\mathcal{K}(X)$}

For any space $X$ we denote by $\mathcal{K}(X)$ the space of all compact subsets of $X$ equipped with the Vietoris topology that is the topology generated by the sets of the form:

$$
V^{-}=\{K \in \mathcal{K}(X): K \subset V\} \quad \text { and } \quad V^{+}=\{K \in \mathcal{K}(X): K \cap V \neq \emptyset\}
$$

where $V$ is an arbitrary open subset of $X$. We recall that if the space $X$ is zerodimensional (compact, Polish) then so is the space $\mathcal{K}(X)$ itself; also if $X$ is perfect then so is $\mathcal{K}^{*}(X)=\mathcal{K}(X) \backslash\{\emptyset\}$. In particular $\mathcal{K}^{*}\left(2^{\omega}\right)$ is homeomorphic to $2^{\omega}$.

\subsection{The Borel structure of the space $\mathcal{F}(X)$}

If the space $X$ is not compact there is no canonical topology on the space $\mathcal{F}(X)$ of all closed subsets of $X$. In this case we will always equip $\mathcal{F}(X)$ with its Effros structure, that is the Borel structure generated by the sets of the form $V^{+}=\{F \in \mathcal{F}(X): F \cap V \neq \emptyset\}$. If $X$ is compact one recovers then the Borel structure generated by the canonical topology of $\mathcal{K}(X)=\mathcal{F}(X)$. It is also well known that if $X$ is Polish then this canonical Borel structure on $\mathcal{F}(X)$ is standard, that is can be generated by some Polish topology. One way of constructing such a topology is to embed the Polish space $X$ in some compact space $\hat{X}$ and then to identify any set $F \in \mathcal{F}(X)$ with its closure $\bar{F}$ in $\hat{X}$. The set $\mathcal{F}(X)$ is then identified to a $\Pi_{2}^{0}$ subset of $\mathcal{K}(\hat{X})$, namely:

$$
\mathcal{F}(X) \approx\{K \in \mathcal{K}(\hat{X}): \overline{K \cap X}=K\} .
$$

This defines on $\mathcal{F}(X)$ a Polish topology which is said to be admissible and one can easily check that the Borel structure defined by an admissible topology is indeed generated by the sets of the form $V^{+}$. Nevertheless, when $X$ is not compact we shall never consider any topological notion when working in $\mathcal{F}(X)$ which will be equipped with its Borel structure solely. Note however that all Borel notions and beyond such as $\boldsymbol{\Sigma}_{1}^{1}, \boldsymbol{\Pi}_{1}^{1}, \boldsymbol{\Sigma}_{2}^{1}, \boldsymbol{\Pi}_{2}^{1}, \cdots$ are meaningful for $\mathcal{F}(X)$.

3.3. We state next the main properties of the space $\mathcal{F}(X)$ when $X$ is Polish, which we will use freely in the sequel:

a) The set $\{(F, G) \in \mathcal{F}(X) \times \mathcal{F}(X): F \subset G\}$ is $\boldsymbol{\Delta}_{1}^{1}$.

b) The set $\{(F, K) \in \mathcal{F}(X) \times \mathcal{K}(X): F \cap K \neq \emptyset\}$ is $\Delta_{1}^{1}$. 
c) The set $\{(F, G) \in \mathcal{F}(X) \times \mathcal{F}(X): F \cap G \neq \emptyset\}$ is $\boldsymbol{\Sigma}_{1}^{1}$.

d) If $A$ is a $\boldsymbol{\Sigma}_{1}^{1}$ subset of $X$ then the $\operatorname{set}\{F \in \mathcal{F}(X): F \cap A \neq \emptyset\}$ is $\boldsymbol{\Sigma}_{1}^{1}$.

e) Let $\mathcal{F}^{*}(X)=\mathcal{F}(X) \backslash\{\emptyset\}$; then there exists a sequence $\left(c_{k}\right)_{k \in \omega}$ of Borel functions $c_{k}: \mathcal{F}^{*}(X) \rightarrow X$ such that for all $F \in \mathcal{F}^{*}(X), c_{k}(F) \in F$ and the set $\left\{c_{k}(F): k \in \omega\right\}$ is dense in $F$.

In fact if we endow $\mathcal{F}(X)$ with some admissible topology then in a) and b) " $\boldsymbol{\Delta}_{1}^{1}$ " can be replaced by " $\boldsymbol{\Pi}_{1}^{0}$ " and in e) "Borel" can be replaced by "first Baire class".

We recall that a compact set valued mapping $\Psi: \Sigma \rightarrow \mathcal{K}(T)$ is said to be u.s.c. if for any open subset $W$ of $T$, the set $\Psi^{-1}\left(W^{-}\right)=\{\alpha \in \Sigma: \Psi(\alpha) \subset W\}$ is an open subset of $\Sigma$. Note that if $T$ is compact this is equivalent to say that the set $\{(\alpha, x) \in \Sigma \times T: x \in \Psi(\alpha)\}$ is closed in $\Sigma \times T$.

The following result will be of constant use in the sequel.

Lemma 3.4. Let $\Sigma$ be a Polish space, $T$ be a compact space, and $\Psi: \Sigma \rightarrow \mathcal{K}(T)$ be an u.s.c. mapping. Let $S$ be a $\Pi_{2}^{0}$ subset of $T$ and $\Phi: \Sigma \rightarrow \mathcal{F}(S)$ be the mapping defined by $\Phi(\alpha)=S \cap \Psi(\alpha)$. If for all $\alpha \in S, \Phi(\alpha)$ is dense in $\Psi(\alpha)$, then the mapping $\Phi$ is Borel.

Proof. Let $V$ be any open subset of $S$. Fix $W$ an open subset of $T$ such that $V=S \cap W$, write $W=\bigcup_{n} F_{n}$ with all the $F_{n}$ 's closed in $T$ and set $W_{n}=T \backslash F_{n}$. It follows then from the density condition that

$$
\begin{aligned}
\Phi(\alpha) \cap V \neq \emptyset & \Longleftrightarrow \Phi(\alpha) \cap W \neq \emptyset \Longleftrightarrow \Psi(\alpha) \cap W \neq \emptyset \\
& \Longleftrightarrow \exists n, \Psi(\alpha) \cap F_{n} \neq \emptyset \Longleftrightarrow \exists n, \Psi(\alpha) \not \subset W_{n} .
\end{aligned}
$$

Hence $\Phi^{-1}\left(V^{+}\right)=\bigcup_{n}\left(\Sigma \backslash \Psi^{-1}\left(W_{n}^{-}\right)\right)$is a $\Sigma_{2}^{0}$ set, which proves that $\Phi$ is Borel.

In all the sequel, given a subset $E$ of a product space $X \times Y$ and an element $x \in X$ in the first factor we set

$$
E(x):=\{y \in Y:(x, y) \in X\} .
$$

\subsection{A family of projective complete sets.}

Let $\Delta \approx 2^{\omega}$ be a copy of the Cantor space and $Q \subset \Delta$ be a countable dense subset of $\Delta$, we define inductively for $n \geq 1$ a pair $\left(\mathbb{P}_{n}(Q, \Delta), \mathbb{Q}_{n}(Q, \Delta)\right)=$ $\left(\mathbb{P}_{n}, \mathbb{Q}_{n}\right)$ of subsets of $\mathcal{K}^{*}\left(\Delta^{n}\right) \approx \mathcal{K}^{*}\left(2^{\omega}\right) \approx 2^{\omega}$ by:

$$
\left\{\begin{array}{l}
\mathbb{P}_{1}=\mathcal{K}^{*}(Q) \\
\mathbb{P}_{n}=\left\{K \in \mathcal{K}\left(\Delta^{n}\right): \forall \alpha \in \Delta, K(\alpha) \in \mathbb{Q}_{n-1}\right\} \quad \text { for } n \geq 2 \\
\mathbb{Q}_{n}=\mathcal{K}^{*}\left(\Delta^{n}\right) \backslash \mathbb{P}_{n}
\end{array}\right.
$$


Hence with the canonical identification $\Delta^{n} \approx \Delta^{n-1} \times \Delta$, for any $K \in \mathcal{K}^{*}\left(\Delta^{n}\right)$ we have:

$$
K \in \mathbb{P}_{n} \Longleftrightarrow \forall \alpha_{1} \in \Delta, \exists \alpha_{2} \in \Delta, \cdots, \exists \alpha_{n-1} \in \Delta, K\left(\alpha_{1}, \cdots, \alpha_{n-1}\right) \subset Q,
$$

if $n \geq 1$ is odd, and

$$
K \in \mathbb{P}_{n} \Longleftrightarrow \forall \alpha_{1} \in \Delta, \exists \alpha_{2} \in \Delta, \cdots, \forall \alpha_{n-1} \in \Delta, K\left(\alpha_{1}, \cdots, \alpha_{n-1}\right) \not \subset Q
$$

if $n$ is even.

It follows then by a straightforward induction that the set $\mathbb{P}_{n}$ is $\boldsymbol{\Pi}_{n}^{1}$ and the set $\mathbb{Q}_{n}$ is $\boldsymbol{\Sigma}_{n}^{1}$. It is also well known that the set $\mathbb{P}_{1}$ is $\boldsymbol{\Pi}_{1}^{1}$-complete.

Lemma 3.6. For all $n \geq 1, \mathbb{P}_{n}$ is $\boldsymbol{\Pi}_{n}^{1}$-complete.

Proof. If $B$ is any $\Pi_{n}^{1}$ subset of $2^{\omega}$ then by definition we can find a sequence $\left(A_{k}, B_{k}\right)_{1 \leq k \leq n}$ satisfying for all $k$ :

1. $B_{n}=B$,

2. $A_{k} \subset 2^{\omega} \times \Delta^{n-k}$ is $\boldsymbol{\Sigma}_{k}^{1}$ and $B_{k}=\left(2^{\omega} \times \Delta^{n-k}\right) \backslash A_{k}$ is $\boldsymbol{\Pi}_{k}^{1}$,

3. $A_{n-k} \subset 2^{\omega} \times \Delta^{k}$ is the projection on $2^{\omega} \times \Delta^{k}$ of $B_{n-k-1} \subset\left(2^{\omega} \times \Delta^{k}\right) \times \Delta$.

Hence $A_{1} \subset C:=2^{\omega} \times \Delta^{n-1}$ is $\Sigma_{1}^{1}$ and setting $P=\Delta \backslash Q \approx \omega^{\omega}$ we can find a closed subset $F$ of $C \times P$ such that $A_{1}$ is the projection of $F$ on $C$. Then if $H$ denotes the closure in $C \times \Delta=2^{\omega} \times \Delta^{n}$ of the set $F$, the mapping $\Phi: \varepsilon \mapsto H(\varepsilon)$ from $2^{\omega}$ to $\mathcal{K}\left(\Delta^{n}\right)$ is u.s.c. hence by Lemma 3.4 the mapping $\Phi$ is Borel, and for all $\varepsilon \in 2^{\omega}$ :

- if $n$ is odd we have:

$$
\begin{aligned}
\varepsilon \in B_{n} & \Longleftrightarrow \forall \alpha_{1} \in \Delta,\left(\varepsilon, \alpha_{1}\right) \in A_{n-1} \\
& \Longleftrightarrow \forall \alpha_{1} \in \Delta, \exists \alpha_{2} \in \Delta:\left(\varepsilon, \alpha_{1}, \alpha_{2}\right) \in B_{n-2} \\
& \Longleftrightarrow \forall \alpha_{1} \in \Delta, \exists \alpha_{2} \in \Delta, \cdots, \exists \alpha_{n-1} \in \Delta, \forall \alpha_{n} \in P,\left(\varepsilon, \alpha_{1}, \cdots, \alpha_{n}\right) \notin F \\
& \Longleftrightarrow \forall \alpha_{1} \in \Delta, \exists \alpha_{2} \in \Delta, \cdots, \exists \alpha_{n-1} \in \Delta, \forall \alpha_{n} \in P,\left(\varepsilon, \alpha_{1}, \cdots, \alpha_{n}\right) \notin H \\
& \Longleftrightarrow \forall \alpha_{1} \in \Delta, \exists \alpha_{2} \in \Delta \cdots, \exists \alpha_{n-1} \in \Delta, H\left(\varepsilon, \alpha_{1}, \cdots, \alpha_{n-1}\right) \subset Q \\
& \Longleftrightarrow H(\varepsilon) \in \mathbb{P}_{n}
\end{aligned}
$$

- similarly if $n$ is even:

$$
\begin{aligned}
\varepsilon \in B_{n} & \Longleftrightarrow \forall \alpha_{1} \in \Delta, \exists \alpha_{2} \in \Delta, \cdots, \forall \alpha_{n-1} \in \Delta, \exists \alpha_{n} \in P:\left(\varepsilon, \alpha_{1}, \cdots, \alpha_{n}\right) \in H \\
& \Longleftrightarrow \forall \alpha_{1} \in \Delta, \exists \alpha_{2} \in \Delta, \cdots, \forall \alpha_{n-1} \in \Delta, H\left(\varepsilon, \alpha_{1}, \cdots, \alpha_{n-1}\right) \not \subset Q \\
& \Longleftrightarrow H(\varepsilon) \in \mathbb{P}_{n}
\end{aligned}
$$

This proves that any $\Pi_{n}^{1}$ subset of $2^{\omega}$ is Borel reducible to $\mathbb{P}_{n}$ hence by Theorem 2.1 the set $\mathbb{P}_{n}$ is $\boldsymbol{\Pi}_{n}^{1}$-complete. 


\section{Connectedness}

We recall that $\mathcal{C}(X)$ denotes the set of all closed connected subsets of $X$. It is well known that if $X$ is compact then $\mathcal{C}(X)$ is a closed, hence compact, subset of $\mathcal{K}(X)$

Proposition 4.1. If $X$ is a Polish space then $\mathcal{C}(X)$ is a $\Pi_{2}^{1}$ subset of $\mathcal{F}(X)$.

Proof. By definition given any $F \in \mathcal{F}(X)$ we have $F \notin \mathcal{C}(X)$ if and only if:

$$
\exists F_{0}, F_{1} \in \mathcal{F}(X): F_{0} \cap F \neq \emptyset, F_{1} \cap F \neq \emptyset, F_{0} \cap F_{1}=\emptyset \text { and } F \subset F_{0} \cup F_{1} .
$$

Since on $\mathcal{F}(X)$ the three arguments relation " $F \subset F_{0} \cup F_{1}$ " is Borel and the binary relation " $F \cap F^{\prime}=\emptyset$ " is $\boldsymbol{\Pi}_{1}^{1}$ it follows that $\mathcal{F}(X) \backslash \mathcal{C}(X)$ is a $\boldsymbol{\Sigma}_{2}^{1}$ set, hence $\mathcal{C}(X)$ is a $\Pi_{2}^{1}$ set.

Theorem 4.2. There exists a Polish space $X \subset \mathbb{I}^{3}$ for which the set $\mathcal{C}(X)$ is $\Pi_{2}^{1}$-complete.

Proof. Fix $\Delta \approx 2^{\omega}$ such that $\left\{\frac{1}{4}, \frac{3}{4}\right\} \subset \Delta \subset\left[\frac{1}{4}, \frac{3}{4}\right]$ and $Q$ a dense countable subset of $\Delta$; then setting $P=\Delta \backslash Q$ we define:

$$
X=\left\{(x, y, z) \in \mathbb{I}^{3}: x \notin \Delta \text { or }(y \in P \text { and } z=0)\right\}
$$

which is clearly a $\Pi_{2}^{0}$ subset of $\mathbb{I}^{3}$ hence a Polish space.

For any set $A \subset \mathbb{I}^{2}$ we set $\tilde{A}=A \times\{0\} \subset \mathbb{I}^{3}$. Let $\Psi: \mathcal{K}^{*}\left(\Delta^{2}\right) \rightarrow \mathcal{K}\left(\mathbb{I}^{3}\right)$ be the mapping defined for all $K \in \mathcal{K}^{*}\left(\Delta^{2}\right)$ by:

$$
\Psi(K)=\left\{u=(x, y, z) \in \mathbb{I}^{3}: z+d(x, \Delta) \geq \frac{1}{2} d(u, \tilde{K})\right\}
$$

where $d$ denotes the standard metric on $\mathbb{I}$ as well as the $\ell^{1}$-metric on $\mathbb{I}^{3}$ :

$$
d\left((x, y, z),\left(x^{\prime}, y^{\prime}, z^{\prime}\right)\right)=\left|x-x^{\prime}\right|+\left|y-y^{\prime}\right|+\left|z-z^{\prime}\right|,
$$

and observe that $\tilde{K} \subset \Psi(K)$. Finally we define $\Phi: \mathcal{K}^{*}\left(\Delta^{2}\right) \rightarrow \mathcal{F}(X)$ by:

$$
\Phi(K)=X \cap \Psi(K) .
$$

Lemma 4.3. The mapping $\Phi$ is Borel.

Proof. We apply Lemma 3.4: observe first that since the set $\{(K, u): u \in \Psi(K)\}$ is a closed subset of $\mathcal{K}\left(\Delta^{2}\right) \times \mathbb{I}^{3}$ then the mapping $\Psi$ is u.s.c. and we now check that for all $K \in \mathcal{K}^{*}\left(\Delta^{2}\right), \Phi(K)$ is dense in $\Psi(K)$.

Let $U$ be an open subset of $\mathbb{I}^{3}$ such that $U \cap \Psi(K) \neq \emptyset$; we have to show that $U \cap \Phi(K) \neq \emptyset$. Fix $u=(x, y, z) \in U \cap \Psi(K)$; we have to find some $u^{\prime}=$ 
$\left(x^{\prime}, y^{\prime}, z^{\prime}\right) \in U \cap \Phi(K)$. For all $t \in \mathbb{I}$ and $\varepsilon>0$ let $\left.J_{t}^{\varepsilon}=\right] t-\frac{\varepsilon}{2}, t+\frac{\varepsilon}{2}[\cap \mathbb{I}$; we may suppose that $U=J_{x}^{\varepsilon} \times J_{y}^{\varepsilon} \times J_{z}^{\varepsilon}$ for some $\varepsilon>0$.

If $x \notin \Delta$ then $u \in \Phi(K)$ and we can take $u^{\prime}=u$. If not then $d(x, \Delta)=0$ and $d((x, y), K)+z=d(u, \tilde{K}) \leq 2 z$ hence $d((x, y), K) \leq z$; and we distinguish three cases:

- If $z<1$ pick $x^{\prime} \in J_{x}^{\varepsilon} \backslash \Delta$, let $\delta<d\left(x^{\prime}, \Delta\right)$ such that $z+\delta<1$ and pick $\left.z^{\prime} \in\right] z, z+\delta\left[\right.$; then $u^{\prime}=\left(x^{\prime}, y, z^{\prime}\right) \in U \cap X, d\left(u^{\prime}, u\right)=\left|x^{\prime}-x\right|+\left|z^{\prime}-z\right| \leq 2 d\left(x^{\prime}, \Delta\right)$ and $d\left(u^{\prime}, \tilde{K}\right) \leq d(u, \tilde{K})+2 d\left(x^{\prime}, \Delta\right) \leq 2 z+2 d\left(x^{\prime}, \Delta\right) \leq 2 z^{\prime}+2 d\left(x^{\prime}, \Delta\right)$; hence $u^{\prime} \in \Phi(K)$.

- If $d((x, y), K)<z=1$ : let $\delta<\varepsilon$ such that $d((x, y), K)<z-\delta$ and pick $x^{\prime} \in J_{x}^{\delta} \backslash \Delta$; then $u^{\prime}=\left(x^{\prime}, y, z\right) \in U \cap X$ and $d\left(u^{\prime}, u\right)=\left|x^{\prime}-x\right| \leq \delta$ hence $d\left(u^{\prime}, \tilde{K}\right) \leq d(u, \tilde{K})+\delta=d((x, y), K)+z+\delta \leq 2 z$ so $u^{\prime} \in \Phi(K)$.

- If $d((x, y), K)=z=1$ : take $\left(x_{0}, y_{0}\right) \in K$ such that $d\left((x, y),\left(x_{0}, y_{0}\right)\right)=1$; then since $\left|y-y_{0}\right| \leq \frac{3}{4}$ we have $\left|x-x_{0}\right| \geq \frac{1}{4}$ and since $x, x_{0} \in \Delta$ we can find $\left.x^{\prime} \in\right] x-\varepsilon, x+\varepsilon\left[\backslash \Delta\right.$ such that $\left|x^{\prime}-x_{0}\right|<\left|x-x_{0}\right|$ and then $u^{\prime}=\left(x^{\prime}, y, 1\right) \in$ $U \cap \Phi(K)$.

Let $\mathbb{P}_{2}=\mathbb{P}_{2}(Q, \Delta)$ (see Section 3.5).

Lemma 4.4. Let $(x, y) \in(\mathbb{I} \backslash \Delta) \times \mathbb{I}$ and set for all $t \in \mathbb{I}$, $u_{t}=(x, y, t)$.

a) If $u_{z} \in \Phi(K)$ then $u_{t} \in \Phi(K)$ for all $t \geq z$.

b) If $K \in \mathbb{P}_{2}$ then there exists $z \in \mathbb{I}$ such that $u_{z} \in \Phi(K)$; in particular $u_{1} \in \Phi(K)$.

Proof. Observe first that since $x \notin \Delta$ then for all $t \in \mathbb{I}, u_{t} \in X \backslash \tilde{K}$ hence

$$
u_{t} \in \Phi(K) \Longleftrightarrow \delta(t):=t+d(x, \Delta)-\frac{1}{2} d\left(u_{t}, \tilde{K}\right) \geq 0 .
$$

a) If $u_{z} \in \Phi(K)$ then for all $t \geq z$ :

$$
\delta(t)-\delta(z)=(t-z)-\frac{1}{2} d\left(u_{t}, u_{z}\right)=(t-z)-\frac{1}{2}(t-z) \geq 0
$$

hence $\delta(t) \geq \delta(z) \geq 0$ and $u_{t} \in \Phi(K)$.

b) Fix $a \in \Delta$ such that $d(x, \Delta)=|x-a|$. Since $K \in \mathbb{P}_{2}$ then there exists $b \in P$ such that $(a, b, 0) \in \tilde{K}$. Then for all $z \geq 1-d(x, \Delta)$ :

$$
d\left(u_{z}, \tilde{K}\right) \leq|x-a|+|y-b|+z \leq d(x, \Delta)+1+z \leq 2 d(x, \Delta)+2 z
$$

hence $\delta(z) \geq 0$; so $u_{z} \in \Phi(K)$ and it follows from part a) that $u_{1} \in \Phi(K)$.

Lemma 4.5. If $K \notin \mathbb{P}_{2}$ then $\Phi(K) \notin \mathcal{C}(X)$. 
Proof. Observe first that for any $K \in \mathcal{K}^{*}\left(\Delta^{2}\right)$ and any $\varepsilon \in\{0,1\}, v_{\varepsilon}=(\varepsilon, 0,1)$ is a member of $\Phi(K)$. Indeed if $w_{\varepsilon}=(a, b, 0) \in \tilde{K}$ is such that $d\left(v_{\varepsilon}, \tilde{K}\right)=d\left(v_{\varepsilon}, w_{\varepsilon}\right)$ then

$$
d\left(v_{\varepsilon}, \tilde{K}\right)=|\varepsilon-a|+|b|+1 \leq \frac{3}{4}+\frac{3}{4}+1=2\left(\frac{1}{4}+1\right)=2(d(\varepsilon, \Delta)+1) .
$$

If $K \notin \mathbb{P}_{2}$ then by definition of $\mathbb{P}_{2}$ there exists some $\alpha \in \Delta$ such that $K(\alpha) \subset Q$ and it follows then from the definition of $X$ that $\Phi(K)(\alpha)=\emptyset$. Hence the sets $V_{0}=\{(x, y, z) \in \Phi(K): x<\alpha\}$ and $V_{1}=\{(x, y, z) \in \Phi(K): x>\alpha\}$ form an open partition of $\Phi(K)$ with $v_{\varepsilon} \in V_{\varepsilon}$, which shows that $\Phi(K)$ is not connected.

Lemma 4.6. If $K \in \mathbb{P}_{2}$ then $\Phi(K) \in \mathcal{C}(X)$.

Proof. Fix $K \in \mathbb{P}_{2}$. Let $\mathcal{J}$ denote the set of all connected components of $\mathbb{I} \backslash \Delta$ and set for all $J \in \mathcal{J}$ :

$$
\Phi_{J}(K)=\Phi(K) \cap\left(J \times \mathbb{I}^{2}\right) .
$$

Claim 1: $\Phi_{J}(K)$ is pathwise connected.

Proof. Set $C_{J}=J \times \mathbb{I} \times\{1\}$. Since $K \in \mathbb{P}_{2}$ then by part b) of Lemma 4.4, $C_{J} \subset \Phi_{J}(K)$ and since it is convex the set $C_{J}$ is pathwise connected. Moreover it follows from part a) of Lemma 4.4 that any element $u=(x, y, z) \in \Phi_{J}(K)$ can be connected in $\Phi_{J}(K)$ by a linear path to the element $u_{1}=(x, y, 1) \in C_{J}$. Hence $\Phi_{J}(K)$ is pathwise connected.

Suppose now that $\left(V_{0}, V_{1}\right)$ is a partition of $\Phi(K)$ into two clopen subsets and that $V_{0}$ contains $v_{0}=(0,0,1)$ and set for $\varepsilon=0,1, \mathcal{J}_{\varepsilon}=\left\{J \in \mathcal{J}: \Phi_{J}(K) \subset V_{\varepsilon}\right\}$. It follows then from Claim 1 that $\mathcal{J}=\mathcal{J}_{0} \cup \mathcal{J}_{1}$.

Claim 2: If $u=(a, b, 0) \in \tilde{K}$ with $a \in \overline{\cup \mathcal{J}_{\varepsilon}}$ then $u \in V_{\varepsilon}$.

Proof. Fix a sequence $\left(J_{k}\right)_{k}$ in $\mathcal{J}_{\varepsilon}$ with for each $k$ some $x_{k} \in J_{k}$ such that $a=\lim _{k} x_{k}$, and let $z_{k}=\left|x_{k}-a\right|$. Since $x_{k} \notin \Delta$ then $u_{k}=\left(x_{k}, b, z_{k}\right) \in X$. Moreover since $u \in \tilde{K}$ then $u \in \Phi(K)$ and

$$
d\left(u_{k}, \tilde{K}\right) \leq d\left(u_{k}, u\right)=\left|x_{k}-a\right|+z_{k}=2 z_{k} \leq 2\left(z_{k}+d\left(x_{k}, \Delta\right)\right)
$$

hence $u_{k} \in \Phi_{J_{k}}(K) \subset V_{\varepsilon}$; and since $V_{\varepsilon}$ is a closed subset of $\Phi(K)$ then $u=$ $\lim _{k} u_{k} \in V_{\varepsilon}$.

Claim 3: $\mathcal{J}=\mathcal{J}_{0}$.

Proof. For otherwise $\mathcal{J}_{1} \neq \emptyset$; let $a=\inf \left(\bigcup \mathcal{J}_{1}\right)$. Since by assumption $v_{0} \in V_{0}$ then $\left[0, \frac{1}{4}\left[\in \mathcal{J}_{0}\right.\right.$ and $\inf J \in \Delta$ for every $J \in \mathcal{J}_{1}$, hence $a \in \Delta$. And since $K \in \mathbb{P}_{2}$, we can find some $b \in P$ such that $u=(a, b, 0) \in \tilde{K} \cap X$. Then fixing for all $k$ some $I_{k} \in \mathcal{J}$ with $\left.I_{k} \cap\right] a-2^{-k}, a\left[\neq \emptyset\right.$, we have that $I_{k} \in \mathcal{J}_{0}$. Also we can find for 
all $k$ some $J_{k} \in \mathcal{J}_{1}$ with $\left.J_{k} \cap\right] a, a+2^{-k}[\neq \emptyset$. It follows then from Claim 2 that $u \in V_{0} \cap V_{1}$ which is a contradiction.

It follows from Claim 3 that $\left((\mathbb{I} \backslash \Delta) \times \mathbb{I}^{2}\right) \cap \Phi(K) \subset V_{0}$. Moreover since $\left(\Delta \times \mathbb{I}^{2}\right) \cap \Phi(K) \subset \tilde{K}$ then it follows from Claim 2 that $\Phi(K) \subset V_{0}$. This proves that $\Phi(K)$ is connected and finishes the proof of Lemma 4.6.

So by Lemma 4.3, Lemma 4.5 and Lemma 4.6 the mapping $\Phi: \mathcal{K}^{*}\left(\Delta^{2}\right) \rightarrow$ $\mathcal{F}(X)$ is Borel and reduces the complete $\Pi_{2}^{1}$ set $\mathbb{P}_{2}$ to $\mathcal{C}(X)$. Hence by Theorem 2.1 the set $\mathcal{C}(X)$ is $\Pi_{2}^{1}$-complete.

\section{Pathwise Connectedness}

We recall that $\mathcal{C}_{\text {path }}(X)$ denotes the set of all closed pathwise connected subsets of $X$.

Proposition 5.1. If $X$ is a Polish space then $\mathcal{C}_{\text {path }}(X)$ is a $\Pi_{2}^{1}$ subset of $\mathcal{F}(X)$.

Proof. Fix a compatible complete metric $d$ on $X$ and equip the space $\Pi(X)$ of all continuous paths $\gamma: \mathbb{I} \rightarrow X$ with the Polish topology of uniform convergence.

Then for any set $F \in \mathcal{F}(X)$ we have:

$$
F \in \mathcal{C}_{\text {path }}(X) \Longleftrightarrow\left\{\begin{array}{l}
\forall x, y \in F, \exists \gamma \in \Pi(X): \gamma(0)=x \\
\gamma(1)=y \text { and } \forall t \in \mathbb{Q}, \gamma(t) \in F
\end{array}\right.
$$

where $\mathbb{Q}$ denotes the set of rational numbers in $\mathbb{I}$.

Since the relations " $x \in F$ " and " $\gamma(t)=x$ " determine relatively Borel, hence $\boldsymbol{\Sigma}_{1}^{1}$, subsets of $X \times \mathcal{F}(X)$ and $\Pi(X) \times \mathbb{I} \times X$ then $\mathcal{C}_{\text {path }}(X)$ is clearly a $\Pi_{2}^{1}$ subset of $\mathcal{F}(X)$.

As pointed out in the Introduction Ajtaj and Becker proved that the $\mathcal{C}_{\text {path }}\left(\mathbb{I}^{n}\right)$ is $\Pi_{2}^{1}$-complete for all $n \geq 3$. In this section we give an alternative proof when $n \geq 4$. This proof relies on some general constructions of pathwise connected spaces that we present first.

Definition 5.2. Given any element $a$ in some set $X$ we set $\mathbb{I}_{a}=\mathbb{I} \times\{a\}$. A set $A \subset \mathbb{I} \times X$ is said to be pinned at $a$ if $A \supset \mathbb{I}_{a}$.

We denote by $\pi_{0}$ and $\pi_{1}$ the projection mappings from $\mathbb{I} \times X$ onto $\mathbb{I}$ and $X$ respectively.

Proposition 5.3. Let $A \subset \mathbb{I} \times X$ be a pinned set at $a \in X$ and suppose that $\pi_{0}\left(A \backslash \mathbb{I}_{a}\right)$ is of empty interior in $\mathbb{I}$. Then $A$ is pathwise connected if and only if for all $\varepsilon \in \mathbb{I}$ the section $A(\varepsilon)$ is pathwise connected. 
Proof. Suppose that all the sections of $A$ are pathwise connected and let $(\alpha, x)$ and $(\beta, y)$ be two elements of $A$. Then $(\alpha, x)$ can be joined to $(\alpha, a)$ by a path in $\{\alpha\} \times A(\alpha) \subset A$, and $(\beta, a)$ can be joined to $(\beta, y)$ by a path in $\{\beta\} \times A(\beta) \subset A$. Since $(\alpha, a)$ can trivially be joined to $(\beta, a)$ by an affine path in $\mathbb{I}_{a}$ then by concatenation, $(\alpha, x)$ can be joined to $(\beta, y)$ by a path in $A$.

Conversely suppose that $A$ is pathwise connected . Let $x$ and $y$ be two elements of some section $A(\varepsilon)$, and let $\gamma$ be a path in $A$ joining $(\varepsilon, x)$ to $(\varepsilon, y)$.

- If $\gamma(\mathbb{I}) \cap \mathbb{I}_{a}=\emptyset$ then $\pi_{0}(\gamma(\mathbb{I}))$ is a connected subset of $\mathbb{I}$ with empty interior, hence it is a singleton, and since $\gamma(0)=(\varepsilon, x)$ then necessarily $\pi_{0}(\gamma(\mathbb{I}))=\{\varepsilon\}$. It follows that $\pi_{1} \circ \gamma$ is a path in $A(\varepsilon)$ joining $x$ to $y$.

- If $\gamma(\mathbb{I}) \cap \mathbb{I}_{a} \neq \emptyset$ let $u=\min \left\{t \in \mathbb{I}: \gamma(t) \in \mathbb{I}_{a}\right\}$ then $\gamma\left(\left[0, u[) \cap \mathbb{I}_{a}=\emptyset\right.\right.$ and as in the previous case we necessarily have $\pi_{0} \circ \gamma(t)=\varepsilon$ for all $t<u$ hence by continuity $\gamma(u)=(\varepsilon, a)$ and it follows that $\gamma_{0}=\pi_{1} \circ \gamma_{\mid[0, u]}$ is a path in $A(\varepsilon)$ joining $x$ to $a$. Similarly if $v=\max \left\{t \in \mathbb{I}: \gamma(t) \in \mathbb{I}_{a}\right\}$ then $\pi_{0} \circ \gamma(t)=\varepsilon$ for all $t \geq v$ and $\gamma_{1}=\pi_{1} \circ \gamma_{\mid[v, 1]}$ is a path in $A(\varepsilon)$ joining $x$ to $a$. Then by the concatenation of $\gamma_{0}$ and $\gamma_{1}$ one gets a path in $A(\varepsilon)$ joining $x$ to $y$.

Proposition 5.4. Let $A, B \subset \mathbb{I} \times X$ be two pinned sets at $a$ and $b$ respectively, and suppose that $E=\pi_{0}\left(A \backslash \mathbb{I}_{a}\right) \cup \pi_{0}\left(B \backslash \mathbb{I}_{b}\right)$ is of empty interior in $\mathbb{I}$.

If $A$ and $B$ are pathwise connected then $C=A \cup B$ is pathwise connected if and only if for some $\varepsilon \in \mathbb{I}$ the section $C(\varepsilon)$ is pathwise connected.

Proof. If $C(\varepsilon)$ is pathwise connected for some $\varepsilon$ then we can find a path in $C$ joining $(\varepsilon, a) \in A$ to $(\varepsilon, b) \in B$, and since $A$ and $B$ are pathwise connected then clearly $C$ is pathwise connected too.

Conversely if $C$ is pathwise connected pick any $\alpha \in \mathbb{I}$, let $\gamma$ be a path in $C$ joining $(\alpha, a)$ to $(\alpha, b)$, consider

$$
u=\max \left\{t \in \mathbb{I}: \gamma(t) \in \mathbb{I}_{a}\right\} \quad \text { and } \quad v=\min \left\{t \in[u, 1]: \gamma(t) \in \mathbb{I}_{b}\right\}
$$

and set $\gamma(u)=\left(\alpha^{\prime}, a\right)$ and $\gamma(v)=\left(\alpha^{\prime \prime}, b\right)$.

- If $u=v$ then $\alpha^{\prime}=\alpha^{\prime \prime}$ and $a=b$ and since by assumption $\pi_{0}\left(C \backslash \mathbb{I}_{a}\right)=E$ is of empty interior in $\mathbb{I}$ then the set $C$ is pinned at $a$ and by Proposition 5.3, $C(\varepsilon)$ is pathwise connected for all $\varepsilon$.

- If $u \neq v$ then $\gamma(] u, v[) \cap\left(\mathbb{I}_{a} \cup \mathbb{I}_{b}\right)=\emptyset$ hence $\pi_{0}(\gamma(] u, v[)) \subset E$ is of empty interior in $\mathbb{I}$, hence as in Proposition $5.3, \pi_{0}(\gamma(] u, v[))$ is a singleton say $\{\varepsilon\}$. It follows by continuity that $\pi_{0}(\gamma([u, v]))=\{\varepsilon\}$ hence $\alpha^{\prime}=\alpha^{\prime \prime}=\varepsilon$ and $\pi_{1} \circ \gamma_{\mid[u, v]}$ is a path in $C(\varepsilon)$ joining $a$ to $b$, and since by Proposition 5.3, $A(\varepsilon)$ and $B(\varepsilon)$ are pathwise connected, then $C(\varepsilon)$ is pathwise connected. 
Remark. Let $X$ be a compact space and suppose that $\Phi: 2^{\omega} \rightarrow \mathcal{K}(X)$ is a continuous mapping reducing some $\Pi_{2}^{1}$ subset $A$ of $2^{\omega}$ to the set $\mathcal{C}_{\text {path }}(X)$. Then since $\mathcal{C}_{\text {path }}(X)$ is a subset of the compact space $\mathcal{C}(X)$ any element from $\bar{A} \backslash A$ is necessarily mapped by $\Phi$ to an element in $\mathcal{C}(X) \backslash \mathcal{C}_{\text {path }}(X)$. Hence Theorem 5.5 below provides implicitly compact sets which are connected but not pathwise connected. As a matter of fact the construction of the space $X$ in this proof will make use of the classical result that if $G$ is the graph of the function $x \mapsto \sin \frac{1}{x}$ then its closure $\bar{G}=G \cup(\{0\} \times[-1,1])$ is connected but not pathwise connected. More precisely, we shall use the fact that no element from $\{0\} \times[-1,1]$ can be joined to an element from $G$ by a path in $\bar{G}$.

Theorem 5.5. (AJTAJ-BECKER) The set $\mathcal{C}_{\text {path }}\left(\mathbb{I}^{4}\right)$ is $\boldsymbol{\Pi}_{2}^{1}$-complete.

Proof. The construction will actually be achieved in the space $\mathbb{I}^{2} \times \mathbb{J}^{2} \approx \mathbb{I}^{4}$ where $\mathbb{J}=[-1,1]$.

Let $\varepsilon \in 2^{\omega}$ that we view as the characteristic function of some set $M_{\varepsilon} \subset \omega$ and set $N_{\varepsilon}=\{0\} \cup M_{\varepsilon}$. Let $\nu_{\varepsilon} \in \omega \leq \omega \backslash\{\emptyset\}$ be the increasing enumeration of $N_{\varepsilon}$ and set:

$$
\left.\left.\left.\left.H_{\varepsilon}^{+}=\bigcup_{j \in \operatorname{dom}\left(\nu_{\varepsilon}\right)}\{(x, y) \in] 2^{-\nu_{\varepsilon}(j+1)}, 2^{-\nu_{\varepsilon}(j)}\right] \times \mathbb{J}: y=2^{-\nu_{\varepsilon}(j)} \sin \frac{\pi}{x}\right\} \subset\right] 0,1\right] \times \mathbb{J}
$$

where by convention $2^{-\nu(i)}=0$ if $i \notin \operatorname{dom}(\nu)$. We also set:

$$
H_{\varepsilon}^{-}=H^{-}=([-1,0] \times\{0\}) \cup(\{0\} \times \mathbb{J}) \subset[-1,0] \times \mathbb{J}
$$

and

$$
H_{\varepsilon}=H_{\varepsilon}^{-} \cup H_{\varepsilon}^{+} \subset \mathbb{J}^{2} .
$$

Observe that for all $\varepsilon, H_{\varepsilon}$ is non empty and compact. More precisely:

- if $N_{\varepsilon}$ is infinite then $\overline{H_{\varepsilon}^{+}}=H_{\varepsilon}^{+} \cup\{(0,0)\}$,

- if $N_{\varepsilon}$ is finite and $k=\max N_{\varepsilon}$ then $\overline{H_{\varepsilon}^{+}}=H_{\varepsilon}^{+} \cup\left(\{0\} \times 2^{-k} \mathbb{J}\right)$.

Let:

$$
\begin{aligned}
& P=\left\{\varepsilon \in 2^{\omega}: \forall m, \exists n \geq m, \varepsilon(n)=1\right\}, \\
& Q=\left\{\varepsilon \in 2^{\omega}: \exists m, \forall n \geq m, \varepsilon(n)=0\right\} .
\end{aligned}
$$

Lemma 5.6. a) For all $\varepsilon, H_{\varepsilon}^{-}$and $H_{\varepsilon}^{+}$are pathwise connected.

b) If $\varepsilon \in P$ then $H_{\varepsilon}$ is pathwise connected.

c) If $\varepsilon \in Q$ then $H_{\varepsilon}$ is not pathwise connected.

Proof. a) For all $\varepsilon, H_{\varepsilon}^{-}=H^{-}$is clearly pathwise connected and $H_{\varepsilon}^{+}$is the graph of a continuous function $\left.\left.f_{\varepsilon}:\right] 0,1\right] \rightarrow \mathbb{J}$ hence also pathwise connected. 
b) If $\varepsilon \in P$ then $\lim _{x \rightarrow 0} f_{\varepsilon}(x)=0$ and $\overline{H_{\varepsilon}^{+}}$is also the graph of a continuous function $\tilde{f}_{\varepsilon}: \mathbb{I} \rightarrow \mathbb{J}$ with $\tilde{f}_{\varepsilon}(0)=0$, hence $\overline{H_{\varepsilon}^{+}}$is pathwise connected. Then writing $H_{\varepsilon}=H^{-} \cup \overline{H_{\varepsilon}^{+}}$each of both sets in this union is pathwise connected and contains the element $(0,0)$. It follows that $H_{\varepsilon}$ is pathwise connected too.

c) If $\varepsilon \in Q$ and $k=\max \left(N_{\varepsilon}\right)$ then $\left.\left.G_{\varepsilon}=H_{\varepsilon} \cap(] 0,2^{-k}\right] \times \mathbb{J}\right)$ is the graph of the function $x \mapsto 2^{-k} \sin \left(\frac{\pi}{x}\right)$ on $\left.] 0,2^{-k}\right]$ and $\overline{G_{\varepsilon}}=G_{\varepsilon} \cup\left(\{0\} \times 2^{-k} \mathbb{J}\right)$. And as pointed out in Remark 5, no element from $\overline{G_{\varepsilon}} \backslash G_{\varepsilon}$ can be joined to an element from $G_{\varepsilon}$ by a path in $\overline{G_{\varepsilon}}$, hence by a path in $H_{\varepsilon}$ since $\overline{G_{\varepsilon}}$ is a retract of $H_{\varepsilon}$.

From now on we identify $2^{\omega}$ to a compact set $\Delta \subset \mathbb{I}$ with endpoints 0 and 1 and set $\mathbb{P}_{1}=\mathbb{P}_{1}(Q, \Delta)$ and $\mathbb{P}_{2}=\mathbb{P}_{2}(Q, \Delta)$.

Let $a=(-1,0)$ and $b=(1,0)$ viewed as elements of $\mathbb{J}^{2}$ so $\mathbb{I}_{a} \cup \mathbb{I}_{b} \subset \mathbb{I} \times \mathbb{J}^{2}$. Observe that for $\varepsilon \in 2^{\omega}$ we have $a \in H_{\varepsilon}^{-}$and $b \in H_{\varepsilon}^{+}$. We then define the mapping $\Psi: \mathcal{K}(\Delta) \rightarrow \mathcal{K}\left(\mathbb{I} \times \mathbb{J}^{2}\right)$ by

$$
\Psi(L)=\mathbb{I}_{a} \cup \mathbb{I}_{b} \cup \bigcup_{\varepsilon \in L}\{\varepsilon\} \times H_{\varepsilon} .
$$

Lemma 5.7. $\Psi(L) \in \mathcal{C}_{\text {path }}\left(\mathbb{I} \times \mathbb{J}^{2}\right)$ if and only if $L \notin \mathbb{P}_{1}$.

Proof. Set $\Psi^{-}(L)=\Psi(L) \cap(\mathbb{I} \times[-1,0] \times \mathbb{J})$ and $\left.\left.\Psi^{+}(L)=\Psi(L) \cap(\mathbb{I} \times] 0,1\right] \times \mathbb{J}\right)$. Then the subsets $\Psi^{-}(L)$ and $\Psi^{+}(L)$ of $\mathbb{I} \times \mathbb{J}^{2}$ are pinned at $a$ and $b$ respectively, and by Lemma 5.6 for all $\varepsilon \in L$ the sets $\Psi^{-}(L)(\varepsilon)=\{a\} \cup H_{\varepsilon}^{-}=H_{\varepsilon}^{-}$and $\Psi^{+}(L)(\varepsilon)=\{b\} \cup H_{\varepsilon}^{+}=H_{\varepsilon}^{+}$are pathwise connected hence by Proposition 5.3, $\Psi^{-}(L)$ and $\Psi^{+}(L)$ are pathwise connected. Since $\Psi(L)=\Psi^{-}(L) \cup \Psi^{+}(L)$ and $\pi_{0}\left(\Psi^{-}(L) \backslash \mathbb{I}_{a}\right) \cup \pi_{0}\left(\Psi^{+}(L) \backslash \mathbb{I}_{b}\right) \subset L$ is of empty interior in $\mathbb{I}$, it follows then from Proposition 5.4 and Lemma 5.6 that:

$$
\begin{aligned}
\Psi(L) \in \mathcal{C}_{\text {path }}\left(\mathbb{I} \times \mathbb{J}^{2}\right) & \Longleftrightarrow \exists \varepsilon \in \mathbb{I}: \Psi(L)(\varepsilon) \in \mathcal{C}_{\text {path }}\left(\mathbb{J}^{2}\right) \\
& \Longleftrightarrow \exists \varepsilon \in L: H_{\varepsilon} \in \mathcal{C}_{\text {path }}\left(\mathbb{J}^{2}\right) \\
& \Longleftrightarrow L \cap \mathbb{P} \neq \emptyset \\
& \Longleftrightarrow L \notin \mathbb{P}_{1} .
\end{aligned}
$$

Let $c=(0, b) \in \mathbb{I} \times \mathbb{J}^{2}$ so $\mathbb{I}_{c} \subset \mathbb{I}^{2} \times \mathbb{J}^{2}$ and observe that for all $L \in \mathcal{K}(\Delta)$, $c \in \Psi(L)$. We then define $\Phi: \mathcal{K}^{*}\left(\Delta^{2}\right) \rightarrow \mathcal{K}\left(\mathbb{I}^{2} \times \mathbb{J}^{2}\right)$ by

$$
\Phi(K)=\mathbb{I}_{c} \cup \bigcup_{\alpha \in \Delta}\{\alpha\} \times \Psi(K(\alpha)) .
$$

Lemma 5.8. $\Phi(K) \in \mathcal{C}_{\text {path }}\left(\mathbb{I}^{2} \times \mathbb{J}^{2}\right)$ if and only if $K \in \mathbb{P}_{2}$. 
Proof. The set $\Phi(K) \subset \mathbb{I} \times\left(\mathbb{I} \times \mathbb{J}^{2}\right)$ is pinned at $c$. It follows then from Proposition 5.3 and Lemma 5.7 that:

$$
\begin{aligned}
\Phi(K) \in \mathcal{C}_{\text {path }}\left(\mathbb{I}^{2} \times \mathbb{J}^{2}\right) & \Longleftrightarrow \forall \alpha \in \mathbb{I}, \Phi(K)(\alpha) \in \mathcal{C}_{\text {path }}\left(\mathbb{I} \times \mathbb{J}^{2}\right) \\
& \Longleftrightarrow \forall \alpha \in \Delta, \Psi(K(\alpha)) \in \mathcal{C}_{\text {path }}\left(\mathbb{I} \times \mathbb{J}^{2}\right) \\
& \Longleftrightarrow \forall \alpha \in \Delta, K(\alpha) \notin \mathbb{P}_{1} \\
& \Longleftrightarrow K \in \mathbb{P}_{2} .
\end{aligned}
$$

To finish observe that the compact-valued mapping $\varepsilon \mapsto H_{\varepsilon}$ is u.s.c. since it has a closed graph. It follows then that $\Psi$ and $\Phi$ viewed as compact-valued mappings are u.s.c. too. In particular the mapping $\Phi: \mathcal{K}^{*}\left(\Delta^{2}\right) \rightarrow \mathcal{K}\left(\mathbb{I}^{2} \times \mathbb{J}^{2}\right)$ is Borel. Then since by Lemma 5.7 and Lemma 5.8, $\Phi$ reduces the $\boldsymbol{\Pi}_{2}^{1}$-complete set $\mathbb{P}_{2}$ to the set $\mathcal{C}_{\text {path }}\left(\mathbb{I}^{2} \times \mathbb{J}^{2}\right)$, it follows from Theorem 2.1 that the set $\mathcal{C}_{\text {path }}\left(\mathbb{I}^{2} \times \mathbb{J}^{2}\right)$ is $\boldsymbol{\Pi}_{2}^{1}$-complete.

\section{Local Connectedness}

We recall that $\mathcal{C}_{\text {loc }}(X)$ denotes the set of all closed locally connected subsets of $X$. We also recall that a topological space is said to be locally connected if any element has a fundamental system of connected (not necessarily open) neighbourhoods. But a basic result asserts that in a locally connected space any element has in fact a fundamental system of connected open neighbourhoods, equivalently that the connected components of any open set are all open.

Proposition 6.1. If $X$ is a compact space then $\mathcal{C}_{\text {loc }}(X)$ is a $\Pi_{3}^{0}$ subset of $\mathcal{K}(X)$. Proof. Fix a countable basis $\left(U_{j}\right)_{j \in \omega}$ of $X$ and let $F \in \mathcal{F}(X)$. If $F$ is locally connected then for all $\varepsilon>0$ and all $x \in F$ there exists an open set $V$ in $X$ such that $x \in V$, $\operatorname{diam} V<\varepsilon$ and $V \cap F$ is connected; then $F^{\prime}=\overline{V \cap F}$ is a closed connected subset of $X$ such that $\operatorname{diam} F^{\prime} \leq \varepsilon$ and if $j$ is such that $x \in U_{j} \subset V$ then $U_{j} \cap F \subset F^{\prime}$. Hence if $F$ is locally connected then

$$
(\star)\left\{\begin{array}{c}
\forall n, \forall x \in F, \exists j \in \omega: x \in U_{j}, \exists F^{\prime} \in \mathcal{C}(X): \\
\operatorname{diam} F^{\prime} \leq 2^{-n} \text { and } U_{j} \cap F \subset F^{\prime}
\end{array}\right.
$$

Conversely if $(\star)$ holds then for all $\varepsilon>0$, any element of $F$ admits a connected neighbourhood of diameter $<\varepsilon$ and so $F$ is locally connected.

Then it follows from the compactness of $F$ that condition $(\star)$ is equivalent to

$$
(\star \star)\left\{\begin{array}{l}
\forall n, \exists J \text { finite } \subset \omega: F \subset \bigcup_{j \in J} U_{j} \text { and } \forall j \in J, \\
\exists F^{\prime} \in \mathcal{C}(X): \operatorname{diam} F^{\prime} \leq 2^{-n} \text { and } U_{j} \cap F \subset F^{\prime}
\end{array}\right.
$$

Hence

$$
\mathcal{C}_{\text {loc }}(X)=\bigcap_{n} \bigcup_{\substack{J \text { finite } \\ J \subset \omega}} \bigcap_{j \in J} \mathcal{K}\left(\bigcup_{j \in J} U_{j}\right) \cap \mathcal{L}_{j, n}
$$


where $\mathcal{L}_{j, n}$ denotes the set of all compact sets $F$ satisfying the second line of $(\star \star)$. Then since $\mathcal{K}\left(\bigcup_{j \in J} U_{j}\right)$ is $\sigma$-compact and each $\mathcal{L}_{j, n}$ is compact as the projection of a closed subset of the compact space $\mathcal{K}(X) \times \mathcal{C}(X)$, it follows that $\mathcal{C}_{\text {loc }}(X)$ is a $\Pi_{3}^{0}$ subset of $\mathcal{K}(X)$.

Theorem 6.2. The set $\mathcal{C}_{\text {loc }}\left(\mathbb{I}^{2}\right)$ is $\boldsymbol{\Pi}_{3}^{0}$-complete.

Proof. For any $a, b \in \mathbb{R}^{2}$ let $[a, b]$ denote the affine segment joining $a$ to $b$. Let $S=[(0,0),(0,1)]$ and given any $\alpha \in \mathbb{I}^{\omega}$ let $S_{n}(\alpha)=\left[(0,0),\left(2^{-n}, \alpha(n)\right)\right]$ and $K(\alpha)=S \cup \bigcup_{n} S_{n}(\alpha)$. Then clearly $K(\alpha)$ is a compact connected subset of $\mathbb{I}^{2}$.

If $\lim _{n} \alpha(n)=0$ then the open subset $K(\alpha) \backslash\{(0,0)\}$ of $K(\alpha)$ is locally homeomorphic to $\mathbb{I}$, hence it is locally connected. Moreover for all $s, t>0$ the open neigbourhood $V_{s, t}=K(\alpha) \cap[0, s[\times[0, t[$ of $\{(0,0)\}$ in $K(\alpha)$ is itself homeomorphic to $K(\alpha)$, so $V_{s, t}$ is connected. Hence $K(\alpha)$ is locally connected.

If $\limsup _{n} \alpha(n)=\delta>0$ then any connected neigbourhood of $(0, \delta)$ in $K(\alpha)$ contains the whole segment $[(0,0),(0, \delta)]$. Hence $K(\alpha)$ is not locally connected.

Since for all $n$ the mapping $\Phi_{n}: \alpha \mapsto S \cup \bigcup_{p \leq n} S_{p}(\alpha)$ is clearly continuous it follows that the uniform limit $\Phi: \alpha \mapsto K(\alpha)$ is continuous too, and by the previous observations $\Phi$ reduces the set $A=\left\{\alpha \in \mathbb{I}^{\omega}: \lim _{n} \alpha(n)=0\right\}$ to the set $\mathcal{C}_{\text {loc }}\left(\mathbb{I}^{2}\right)$, and it is well known that the set $A$ is $\Pi_{3}^{0}$-complete.

We recall that $\mathcal{A}\left(\boldsymbol{\Pi}_{1}^{1}\right)$ denotes the class of all sets obtained by the Souslin $\mathcal{A}$-operation from $\boldsymbol{\Pi}_{1}^{1}$ sets, and $\check{\mathcal{A}}\left(\boldsymbol{\Pi}_{1}^{1}\right)$ its dual class.

Theorem 6.3. If $X$ is a Polish space then $\mathcal{C}_{\text {loc }}(X)$ is a $\check{\mathcal{A}}\left(\boldsymbol{\Pi}_{1}^{1}\right)$ subset of $\mathcal{F}(X)$.

Proof. Fix a sequence $\left(c_{k}\right)_{k \in \omega}$ of Borel functions $c_{k}: \mathcal{F}^{*}(X) \rightarrow X$ such that for all $F \in \mathcal{F}^{*}(X), c_{k}(F) \in F$ and the set $\left\{c_{k}(F): k \in \omega\right\}$ is dense in $F$ (see Section 3). Then given any $F \in \mathcal{F}^{*}(X)$ and any $V$ open subset of $X$ we define for all $k$ :

$$
C_{k}(F, V)=\overline{\left\{x: \exists K \in \mathcal{C}(X) \text { compact }:\left\{x, c_{k}(F)\right\} \subset K \subset F \cap V\right\}}
$$

which is clearly a closed connected subset of $F$ containing $c_{k}(F)$.

Claim 1: The set $\mathcal{A}_{k}=\left\{(x, F) \in X \times \mathcal{F}^{*}(X): x \in C_{k}(F, V)\right\}$ is $\boldsymbol{\Sigma}_{1}^{1}$.

Proof. Let $\hat{X}$ be any compactification of $X$ and $\hat{U}_{i}$ be open subsets of $\hat{X}$ such that $U_{i}=X \cap \hat{U}_{i}$; then

$$
x \in C_{k}(F, V) \Longleftrightarrow \forall i, x \notin U_{i} \text { or } \exists K \in \mathcal{C}(\hat{X}):\left\{\begin{array}{l}
K \cap \hat{U}_{i} \neq \emptyset, K \subset F \cap V, \\
x \in K, c_{k}(F) \in K
\end{array}\right.
$$

Then observe that $\hat{U}_{i}$ being open and $V$ being a $\Pi_{2}^{0}$ subset of the compact space $\hat{X}$ the conditions " $K \in \mathcal{C}(\hat{X}), K \cap \hat{U}_{i} \neq \emptyset, K \subset F \cap V$ " define a $\Pi_{2}^{0}$ subset of 
the compact space $\mathcal{K}(\hat{X})$. Also since $c_{k}$ is Borel then the condition " $c_{k}(F) \in K$ " determines a Borel, hence a $\boldsymbol{\Sigma}_{1}^{1}$, subset of $\mathcal{F}^{*}(X) \times \mathcal{K}(\hat{X})$. It follows that $\mathcal{A}_{k}$ is $\Sigma_{1}^{1}$

Claim 2: For any open set $U \subset V$, the set $\left\{F \in \mathcal{F}^{*}(X): F \cap U \subset C_{k}(F, V)\right\}$ is $\Sigma_{1}^{1}$.

Proof. Let $J=\left\{j \in \omega: \overline{U_{j}} \subset U\right\}$; then $U=\bigcup_{j \in J} U_{j}$ and

$$
F \cap U=\bigcup_{j \in J} F \cap U_{j} \subset \bigcup_{j \in J} \overline{F \cap U_{j}} \subset \bigcup_{j \in J} F \cap \overline{U_{j}}=F \cap U
$$

hence, since $\left\{c_{i}(F): i \in \omega\right\} \cap U_{j}$ is dense in $F \cap U_{j}$ :

$$
\begin{aligned}
F \cap U \subset C_{k}(F, V) & \Longleftrightarrow \forall j \in J, \overline{F \cap U_{j}} \subset C_{k}(F, V) \\
& \Longleftrightarrow \forall j \in J, \forall i \in \omega, c_{i}(F) \notin U_{j} \text { or } c_{i}(F) \in C_{k}(F, V) .
\end{aligned}
$$

The conclusion follows then from Claim 1.

Consider the tree

$$
T=\left\{s \in \omega^{<\omega}: \forall m<|s|, \operatorname{diam}\left(U_{s(m)}\right) \leq 2^{-m} \text { and } \overline{U_{s(m)}} \subset U_{s(m-1)}\right\}
$$

Set $\mathcal{E}_{\emptyset}=\mathcal{F}^{*}(X)$ and for all $s \in T \cap \omega^{n+1}$ let

$$
\mathcal{E}_{s}=\left\{F \in \mathcal{F}^{*}(X): F \cap U_{s(n)} \neq \emptyset \text { and } \forall k, F \cap U_{s(n)} \not \subset C_{k}\left(F, U_{s(0)}\right)\right\}
$$

Claim 3: $\mathcal{F}^{*}(X) \backslash \mathcal{C}_{\text {loc }}(X)=\mathcal{A}\left(\left(\mathcal{E}_{s}\right)_{s \in T}\right)$.

Proof. If $F$ is not locally connected we can find some $U_{i_{0}}$ and $x \in F \cap U_{i_{0}}$ such that $F \cap U_{i_{0}}$ does not contain any connected neigbourhood of $x$ in $F$. We can also find an infinite branch $\sigma$ of $T$ such that $\sigma(0)=i_{0}$ and $x \in U_{\sigma(n)}$ for all $n$. Then for all $k$, since $C_{k}\left(F, U_{i_{0}}\right)$ is a connected subset of $F \cap U_{\sigma(0)}$, then for no $n$ we can have $F \cap U_{\sigma(n)} \subset C_{k}\left(F, U_{i_{0}}\right)$. Hence $F \in \mathcal{E}_{\sigma_{\mid n}}$ for all $n$, and so $F \in \mathcal{A}\left(\left(\mathcal{E}_{s}\right)_{s \in T}\right)$.

Conversely if for some infinite branch $\sigma$ of $T$, we have $F \in \mathcal{E}_{\sigma_{\mid n}}$ for all $n$ then $F$ is not locally connected. For otherwise let $\{x\}=\bigcap_{n} U_{\sigma(n)}$ and let $C$ be the connected component of $x$ in $F \cap U_{\sigma(0)}$. Since $F$ is assumed to be locally connected then $C$ is open in $F$ and we can find some $m$ and then some $k$ such that $c_{k}(F) \in F \cap U_{\sigma(m)} \subset C$. Moreover $C$ is a connected and locally connected Polish space, and it is well known that such a space is actually pathwise connected. Hence any element in $C$ can be joined to $c_{k}(F)$ by a path in $C \subset F \cap U_{\sigma(0)}$. It follows that $x \in F \cap U_{\sigma(m)} \subset C_{k}\left(F, U_{\sigma(0)}\right)$ which contradicts that $F \in \mathcal{E}_{\sigma_{\mid m+1}}$.

To finish the proof of Theorem 6.1 observe that by Claim 2 each $\mathcal{E}_{s}$ is a $\Pi_{1}^{1}$ set hence by Claim 3 the set $\mathcal{C}_{\text {loc }}(X)$ is in the class $\check{\mathcal{A}}\left(\boldsymbol{\Pi}_{1}^{1}\right)$. 
Theorem 6.4. There exists a Polish space $X \subset \mathbb{I}^{3}$ for which the set $\mathcal{C}_{\text {loc }}(X)$ is $\check{\mathcal{A}}\left(\boldsymbol{\Pi}_{1}^{1}\right)$-complete.

Proof. The space $X$ will be of the form:

$$
X=\bigcup_{s \in \omega<\omega} X_{s}
$$

where each $X_{s}$ for $s \neq \emptyset$, is contained in a 2-dimensional affine space and is affinely isomorphic to a fixed Polish space $Y \subset \mathbb{J} \times \mathbb{J}$ of the plane. The proof is rather technical and will be split in three parts:

a) Definition and study of the space $Y$.

b) Definition of the space $X$.

c) Construction for any given $\mathcal{A}\left(\boldsymbol{\Pi}_{1}^{1}\right)$ set $A \subset 2^{\omega}$ of a Borel mapping $\Phi: 2^{\omega} \rightarrow \mathcal{F}^{*}(X)$ which reduces $A$ to $\mathcal{F}(X) \backslash \mathcal{C}_{\text {loc }}(X)$.

a) Definition of the space $Y$ : We fix $\left\{-\frac{1}{2}, \frac{1}{2}\right\} \subset \Delta \subset\left[-\frac{1}{2}, \frac{1}{2}\right]$ a copy of $2^{\omega}$ such that $Q:=\Delta \cap \mathbb{Q}$ is dense in $\Delta$, and set $\mathbb{P}_{1}=\mathbb{P}_{1}(Q, \Delta) \subset \mathcal{K}^{*}(\Delta)$.

Let $a=(0,1), a^{*}=(0,-1), b=(1,0), b^{*}=(-1,0)$ and let $\Sigma \subset \mathbb{J} \times \mathbb{J}$ be the full square of vertices $a, a^{*}, b, b^{*}$, that is the convex hull of the set $\left\{a, a^{*}, b, b^{*}\right\}$. We point out here that despite the formal symmetry between $a, a^{*}$ and $b, b^{*}$ the central role in the construction will be played by $a, a^{*}$. We then define

$$
Y=\Sigma \backslash(Q \times\{0\})
$$

which is clearly a $\Pi_{2}^{0}$ subset of $\mathbb{J} \times \mathbb{J}$.

In this part of the proof by square we shall mean a subsquare of $\Sigma$ whose edges are parallel to the edges of $\Sigma$. Hence if we consider the convex cone

$$
C=\left\{(x, y) \in \mathbb{R}^{2}: y \geq|x|\right\}
$$

then a square is a set of the form $(v+C) \cap(w-C)$ where $(v, w)$ is a pair of elements of $\Sigma$ with same first coordinate. Note that any element $u \in \Sigma$ admits a basis of neighbourhoods in $\Sigma$ constituted by squares with vertices $v, w \in \Sigma \backslash(\mathbb{J} \times\{0\})$.

For any $E \subset \mathbb{R}^{2}$ we set:

$$
E^{0}=E \cap(\mathbb{R} \times\{0\}), \quad E^{+}=E \cap(\mathbb{R} \times] 0,+\infty[), \quad E^{-}=E \cap(\mathbb{R} \times]-\infty, 0[),
$$

so

$$
E=E^{0} \cup E^{+} \cup E^{-}
$$

Lemma 6.5. There exists a Borel mapping $\Psi: \mathcal{K}^{*}(\Delta) \rightarrow \mathcal{F}(Y)$ such that for all $L \in \mathcal{K}^{*}(\Delta)$, 
a) $\Psi(L)$ is locally connected; moreover for any convex set $W$ containing a (resp. $a^{*}$ ) the set $W^{+} \cap \Psi(L)$ (resp. $W^{-} \cap \Psi(L)$ ) is connected.

b) if $L \notin \mathbb{P}_{1}$ then $\Psi(L) \in \mathcal{C}(Y)$.

c) if $L \in \mathbb{P}_{1}$ then a and $a^{*}$ are separated in $\Psi(L)$ by a clopen set, hence $\Psi(L) \notin$ $\mathcal{C}(Y)$.

Proof. Let $\hat{\Psi}: \mathcal{K}^{*}(\Delta) \rightarrow \mathcal{K}(\Sigma)$ and $\Psi: \mathcal{K}^{*}(\Delta) \rightarrow \mathcal{F}(Y)$ defined for all $L \in \mathcal{K}^{*}(\Delta)$ by:

$$
\hat{\Psi}(L)=\{(x, y) \in \Sigma:|y| \geq d(x, L)\} \supset L \times\{0\}
$$

where $d$ is the standard metric on $\mathbb{I}$, and

$$
\Psi(L)=\hat{\Psi}(L) \cap Y .
$$

The mapping $\hat{\Psi}$ is clearly u.s.c. and for all $L \in \mathcal{K}^{*}(\Delta), \Psi(L)$ is dense in $\hat{\Psi}(L)$ hence by Lemma 3.4 the mapping $\Psi$ is Borel.

We then set for $\varepsilon=0,+,-$ :

$$
\Psi^{\varepsilon}(L)=\Psi(L) \cap Y^{\varepsilon}
$$

and for all $\alpha \in \mathbb{J}$ :

$$
\begin{aligned}
& C_{\alpha}=\left\{(x, y) \in \mathbb{R}^{2}: y \geq|x-\alpha|\right\}=(\alpha, 0)+C \\
& C_{\alpha}^{\prime}=\left\{(x, y) \in \mathbb{R}^{2}: y \leq-|x-\alpha|\right\}=(\alpha, 0)-C .
\end{aligned}
$$

Hence

$$
\Psi^{+}(L)=\bigcup_{\alpha \in L} C_{\alpha} \cap \Sigma^{+} \quad \text { and } \quad \Psi^{-}(L)=\bigcup_{\alpha \in L} C_{\alpha}^{\prime} \cap \Sigma^{-} .
$$

The inclusions from right to left are obvious since for all $x, \alpha, d(x, L) \leq|x-\alpha|$, and from left to right since for all $x, d(x, L)=|x-\alpha|$ for some $\alpha$.

a) Let $u \in \Psi(L)$ and $\varepsilon>0$. Consider a square $V=(v+C) \cap(w-C)$ such that $u \in V$ with $\operatorname{diam}(V)<\varepsilon$. By symmetry we may suppose that $u \notin \Psi^{-}(L)$ and $w \in \Sigma^{+}$.

Since the cones $C_{\alpha}$ and $w-C$ have parallel edges then $V \cap C_{\alpha} \neq \emptyset$ if and only if $w \in C_{\alpha}$, and since $w \in \Sigma^{+}$then

$$
V \cap \Psi^{+}(L)=\bigcup\left\{V \cap C_{\alpha} \cap \Sigma^{+}: w \in C_{\alpha}\right\} .
$$

Note that each $V \cap C_{\alpha} \cap \Sigma^{+}$is a convex, hence connected, set and since in the formula above all the sets $V \cap C_{\alpha} \cap \Sigma^{+}$have $w$ as a common element then $V \cap \Psi^{+}(L)$ is connected. We now distinguish two cases: 
- If $u \in \Psi^{+}(L)$ then we can choose $V$ small enough so that $V \cap \Psi(L)=$ $V \cap \Psi^{+}(L)$ and we are done.

- If $u \in \Psi^{0}(L)$ then $\left(V \cap \Psi^{+}(L)\right) \cup\left(V \cap \Psi^{0}(L)\right)={\overline{V \cap \Psi^{+}(L)}}^{Y}$ is connected, and we necessarily have that $v \in \Sigma^{-}$hence $\left(V \cap \Psi^{-}(L)\right) \cup\left(V \cap \Psi^{0}(L)\right)$ is connected too, and since $V \cap \Psi^{0}(L) \neq \emptyset$ it follows that $V \cap \Psi(L)$ is connected.

Finally if $W$ is any convex set containing $a$ then for any $x \in Z=W^{+} \cap \Psi(L)$ the segment $[a, x]$ is contained in $Z$ hence $Z$ is connected.

b) Recall that $\Psi^{+}(L)=\bigcup_{\alpha \in L} C_{\alpha} \cap \Sigma^{+}$and the sets $C_{\alpha} \cap \Sigma^{+}$are all convex hence connected; and since $a=(0,1) \in C_{\alpha} \cap \Sigma^{+}$for all $\alpha$ then $\Psi^{+}(L)$ is connected. Similarly $\Psi^{-}(L)$ is connected. Then as above $\Psi^{+}(L) \cup \Psi^{0}(L)={\overline{\Psi^{+}(L)}}^{Y}$ and $\Psi^{-}(L) \cup \Psi^{0}(L)=\bar{\Psi}^{-(L)} Y$ are connected. Hence if $L \notin \mathbb{P}_{1}$ then $\Psi^{0}(L) \neq \emptyset$ and $\Psi(L)=\Psi^{0}(L) \cup \Psi^{+}(L) \cup \Psi^{-}(L)$ is connected.

c) If $L \in \mathbb{P}_{1}$ then $\Psi^{0}(L)=\emptyset$ and then $\left(\Psi^{+}(L), \Psi^{-}(L)\right)$ form a non trivial open covering of $\Psi(L)$ separating $a$ and $a^{*}$.

This finishes the proof of Lemma 6.5.

\section{Some variations of the space $Y$ :}

We recall that $a=(0,1), a^{*}=(0,-1), b=(1,0), b^{*}=(-1,0)$ and $\Sigma \subset \mathbb{J} \times \mathbb{J}$ is the full square of vertices $a, a^{*}, b, b^{*}$.

For any pair $\left(u^{*}, u\right)$ of distinct elements in $\mathbb{R}^{3}$ and any $\varepsilon>0$ let $h: \mathbb{R}^{2} \rightarrow \mathbb{R}^{3}$ be the unique affine mapping such that

$h(a)=u, \quad h\left(a^{*}\right)=u^{*}, \quad h(b)=\frac{u+u^{*}}{2}+\left(0,0, \frac{\varepsilon}{2}\right), \quad h\left(b^{*}\right)=\frac{u+u^{*}}{2}-\left(0,0, \frac{\varepsilon}{2}\right)$.

Set $\Sigma^{\left(u^{*}, u, \varepsilon\right)}=h(\Sigma), \Sigma_{+}^{\left(u^{*}, u, \varepsilon\right)}=h\left(\Sigma^{+}\right), \Sigma_{-}^{\left(u^{*}, u, \varepsilon\right)}=h\left(\Sigma^{-}\right), Y^{\left(u^{*}, u, \varepsilon\right)}=h(Y)$ and let $\left.\Psi^{\left(u^{*}, u, \varepsilon\right)}: \mathcal{K}^{*}(\Delta) \rightarrow \mathcal{F}\left(Y^{\left(u^{*}, u, \varepsilon\right.}\right)\right)$ be the mapping defined by:

$$
\Psi^{\left(u^{*}, u, \varepsilon\right)}(L)=h(\Psi(L))
$$

where $\Psi$ is the mapping given by Lemma 6.5 .

b) Definition of the space $X$ :

For any $s \in \omega^{\leq \omega}$ let $s^{+}$be the element of $(\omega \backslash\{0\})^{\leq \omega}$ of the same length such that $s^{+}(i)=s(i)+1$ for all $i<\left|s^{+}\right|=|s|$, let $\chi(s) \in \mathbb{I}$ be the real whose expansion into continued fraction is given by $s^{+}$and let $I_{s}$ denote the closed interval of endpoints $\chi(s)$ and $\chi(s \frown\langle 0\rangle)$. We recall that:

1. $\chi(\emptyset)=0, \chi(\langle n\rangle)=\frac{1}{n+1}$ and $\chi(s \frown\langle n, 0\rangle)=\chi(s \frown\langle n+1\rangle)$;

2. $\chi(s)$ is a rational number if and only if the sequence $s$ is finite; 
3. for all $s \prec t, \chi(t) \in I_{t} \subset I_{s}$;

4. $I_{s \frown\langle n\rangle} \cap I_{s \frown\langle n+1\rangle}=\{\chi(s \frown\langle n\rangle)\}$ and $I_{s \frown\langle n\rangle} \cap I_{s \frown\langle m\rangle}=\emptyset$ if $|m-n|>1$;

5. $\lim \sup _{n \rightarrow \infty} I_{s \frown\langle n\rangle}=\{\chi(s)\}$.

For any $s \in \omega^{<\omega}$ we set $\nu(s)=|s|+\sum_{k<|s|} s(k)$ and let:

$$
\varepsilon_{s}=2^{-\nu(s)} \quad ; \quad a_{s}=\left(\chi(s), \varepsilon_{s}, 2^{-|s|}\right)
$$

and if $s \neq \emptyset$ we set $s^{*}=s_{|| s \mid-1}$ and let

$$
\begin{gathered}
\Sigma_{s}=\Sigma^{\left(a_{s^{*}}, a_{s}, \varepsilon_{s}\right)} \quad ; \quad \Sigma_{s}^{+}=\Sigma_{+}^{\left(a_{s^{*}}, a_{s}, \varepsilon_{s}\right)} \quad ; \quad \Sigma_{s}^{-}=\Sigma_{-}^{\left(a_{s^{*}}, a_{s}, \varepsilon_{s}\right)} \\
X_{s}=Y^{\left(a_{s^{*}}, a_{s}, \varepsilon_{s}\right)} ; \quad N_{s}=I_{s^{*}} \times\left[0, \varepsilon_{s^{*}}\right] \times\left[0,2^{-\left|s^{*}\right|}\right] \\
\Psi_{s}=\Psi^{\left(a_{s^{*},}, a_{s}, \varepsilon_{s}\right)}: \mathcal{K}^{*}(\Delta) \rightarrow \mathcal{F}\left(X_{s}\right) .
\end{gathered}
$$

Observe that if $\chi(s)=\chi(t)$ and $|s|=|t|$ then $s=t$, so $a_{s} \neq a_{t}$ whenever $s \neq t$.

Finally for any set $E \subset \mathbb{R}$ we set $\tilde{E}:=E \times\{0\} \times\{0\}$ and define

$$
X_{\emptyset}=\tilde{P} \quad \text { and } \quad X=\bigcup_{s \in \omega<\omega} X_{s} .
$$

One can then check the following properties which follow from the definitions and properties $(i)$ to $(v)$ above:

1. for all $t \succeq s, a_{t} \in X_{t} \subset \Sigma_{t} \subset N_{t} \subset N_{s}$;

2. for all $t \neq s, \Sigma_{s} \cap \Sigma_{t} \neq \emptyset$ if and only if $\Sigma_{s} \cap \Sigma_{t}=\left\{a_{r}\right\}$ with: $r=s=t^{*}$ or $r=t=s^{*}$ or $r=s^{*}=t^{*}$;

3. $\lim _{n \rightarrow \infty} a_{s \frown\langle n\rangle}=\hat{a}_{s}:=\left(\chi(s), 0,2^{-|s|-1}\right)$;

4. $\lim \sup _{n \rightarrow \infty} \Sigma_{s \frown\langle n\rangle}=\liminf _{n \rightarrow \infty} X_{s \frown\langle n\rangle}=\hat{\Sigma}_{s}$ is the line segment $\left[\hat{a}_{s}, a_{s}\right]$;

5. $\lim \sup _{n \rightarrow \infty} \bigcup_{t \succ s\urcorner\langle n\rangle} \Sigma_{t}=\tilde{\Sigma}_{s}:=\left\{(\chi(s), 0, z): 0 \leq z \leq 2^{-|s|-1}\right\} ;$

6. $\hat{\Sigma}_{s} \cap X=\left\{a_{s}\right\}$ and $\tilde{\Sigma}_{s} \cap X=\emptyset$.

Lemma 6.6. $X$ is a Polish space.

Proof. Observe first that $X_{\emptyset}=\tilde{P}=X \cap \tilde{\mathbb{I}}$ is closed in $X$. Consider now a sequence $\left(u_{k}\right)_{k}$ in $X$ converging to $u=(x, y, z)$ with for all $k, u_{k} \in X_{s_{k}}$ for some $s_{k} \neq \emptyset$. Then up to the extraction of some subsequence we may suppose that:

- either there exists $s \in \omega^{<\omega}$ such that $s_{k}=s$ for all $k$, and then $u \in X_{s}$; 
- or there exists $s \in \omega^{<\omega}$ such for all $k, s_{k} \supset s^{\frown}\left\langle n_{k}\right\rangle$ with $\lim _{k \rightarrow \infty} n_{k}=\infty$, and then by conditions (iv) and (v), $u \in \hat{\Sigma}_{s} \cup \tilde{\Sigma}_{s}$, hence by property (vi) either $u \notin X$ or $u=a_{s}$;

- or there exists an infinite sequence $\sigma \in \omega^{\omega}$ such for all $k, s_{k} \supset \sigma_{\mid \ell_{k}}$ with $\lim _{k \rightarrow \infty} \ell_{k}=\infty$. It follows then from property (i) that $u=\lim u_{k}=(\chi(\sigma), 0,0) \in$ $\tilde{P}$.

Hence if $\bar{X}$ denotes the closure of $X$ in $\mathbb{I}^{3}$ then

$$
\bar{X} \backslash X=\bigcup_{s}\left(\tilde{\Sigma}_{s} \cup \hat{\Sigma}_{s} \backslash\left\{a_{s}\right\}\right)
$$

which is a countable union of compact sets, hence $X$ is a $\Pi_{2}^{0}$ subset of $\mathbb{I}^{3}$.

c) Construction of the reduction mappings:

We now fix an arbitrary regular Souslin system $\left(A_{s}\right)_{s \in \omega}<\omega$ of $\Pi_{1}^{1}$-sets and let $A=\mathcal{A}\left(\left(A_{s}\right)_{s \in \omega<\omega}\right)$ the result of the Souslin operation on this system. Since $\mathbb{P}_{1}$ is $\Pi_{1}^{1}$-complete we can fix for all $\sigma \in \omega^{<\omega}$, a continuous mapping $\psi_{s}: 2^{\omega} \rightarrow \mathcal{K}^{*}(\Delta)$ which reduces $A_{s}$ to $\mathbb{P}_{1}$, and set $\varphi_{s}=\Psi_{s} \circ \psi_{s}$ for $s \neq \emptyset$. So by Lemma 6.5 , $\varphi_{s}: 2^{\omega} \rightarrow \mathcal{F}^{*}\left(X_{s}\right)$ is a Borel mapping satisfying for all $\alpha$ :

a) $\varphi_{s}(\alpha)$ is locally connected and for any convex set $W$ containing $a_{s}$ the set $W \cup \Sigma_{s}^{+} \cap \varphi_{s}(\alpha)$ is connected (and similarly for $a_{s^{*}}$ and $\Sigma_{s}^{-}$).

b) if $\alpha \notin A_{s}$ then $\varphi_{s}(\alpha) \in \mathcal{C}\left(X_{s}\right)$.

c) if $\alpha \in A_{s}$ then $a_{s}$ and $a_{s}^{*}$ are separated by a clopen set, hence $\varphi_{s}(\alpha) \notin$ $\mathcal{C}\left(X_{s}\right)$.

We finally define for all $\alpha \in 2^{\omega}$

$$
\Phi(\alpha)=\tilde{P} \cup \bigcup_{\substack{s \in \omega<\omega \\ s \neq \emptyset}} \varphi_{s}(\alpha) .
$$

Lemma 6.7. $\Phi(\alpha) \in \mathcal{F}^{*}(X)$.

Proof. Consider as in the proof of Lemma 6.6 a sequence $\left(u_{k}\right)_{k}$ in $\Phi(\alpha)$ converging to some $u \in X$. Again since $\tilde{P}$ is closed in $\Phi(\alpha)$ we may suppose that for all $k$, $u_{k} \in \varphi_{s_{k}}(\alpha)$ for some $s_{k} \neq \emptyset$. Then repeating the arguments of Lemma 6.6 we are led to three alternatives. In the first alternative we are reduced to the case where $s_{k}=s$ for all $k$, and then $u_{k} \in \varphi_{s}(\alpha)$ for all $k$, hence $u \in \varphi_{s}(\alpha) \subset \Phi(\alpha)$. In the two other alternatives we have $u \in\left(\tilde{\Sigma}_{s} \cup \hat{\Sigma}_{s}\right) \cap X$ hence $u=a_{s}$, or $u \in \tilde{P}$, and in both cases $u \in \Phi(\alpha)$.

Lemma 6.8. $\Phi(\alpha)$ is locally connected at any element of $\Phi(\alpha) \backslash \tilde{P}$. 
Proof. Observe that by property (iv) the set $F:=\left\{a_{s}: s \in \omega^{<\omega}\right\}$ is a closed subset of $\Phi(\alpha)$, and it follows from properties (iv), (v) and (vi) that for all $s$ in $S^{*}=\omega^{<\omega} \backslash\{\emptyset\}$ the set $\Sigma_{s} \backslash\left\{a_{s}, a_{s^{*}}\right\}$ is open in $X$ hence $U_{s}:=\varphi_{s}(\alpha) \backslash\left\{a_{s}, a_{s^{*}}\right\}$ is an open subset of $\Phi(\alpha)$, and by the very choice of $\varphi_{s}$ the set $U_{s}$ is locally connected. It follows that $U:=\Phi(\alpha) \backslash(\tilde{P} \cup F)=\bigcup_{s \in S^{*}} U_{s}$ is a locally connected open subset of $\Phi(\alpha)$. So $\Phi(\alpha)$ is locally connected at any element of $U$.

If $u=a_{s} \in F$ for some $s \in \omega^{<\omega}$ then the set

$$
S=\left\{r \in \omega^{<\omega}: r \neq s \text { and } \nu(r) \leq 1+\nu(s)\right\}
$$

is finite and $u \notin \hat{\Sigma}_{r}$ for all $r \in S$. It follows that the open neighbourhood $V=$ $\left\{(x, y, z) \in \mathbb{I}^{2} \times \mathbb{R}: y>\frac{\varepsilon_{s}}{2}\right\}$ of $u$ meets only finitely many segments $\left[a_{r}, \hat{a}_{r}\right]$ with $r \in S$ and that $u$ does not belong to $\left.F_{u}=\overline{\bigcup\left\{\Sigma_{r}: r^{*} \in S, r \neq s\right.}\right\}$. So one can find a convex neighbourhood $W \subset V$ of $u$ such that $W \cap F_{u}=\emptyset$ and that $(x, y, z) \in W \Longrightarrow \frac{3 \varepsilon_{s}}{4}<y<\frac{5 \varepsilon_{s}}{4}$ whence $\Sigma_{t} \cap W \neq \emptyset \Longrightarrow u \in \Sigma_{t}$, that is $t=s$ or $t^{*}=s$ and moreover $\Psi_{s}^{-1}(W) \subset \Sigma^{+}$and $\Psi_{s^{\frown}\langle n\rangle}^{-1}(W) \subset \Sigma^{-}$.

Hence $W \cap \Phi(\alpha)=\bigcup\left\{W \cap \varphi_{t}(\alpha): t=s\right.$ or $\left.t^{*}=s\right\}$ and it follows from property a) stated above that $W \cap \varphi_{t}(\alpha)$ is connected and contains $u$ for such a $t$, which proves that $W \cap \Phi(\alpha)$ is connected, hence that $\Phi(\alpha)$ is locally connected at $u$.

Lemma 6.9. If $\alpha \notin A$ then $\Phi(\alpha) \in \mathcal{C}_{\text {loc }}(X)$.

Proof. By Lemma 6.8 we only need to prove that $\Phi(\alpha)$ is locally connected at any element of $\tilde{P}$. So let $\varepsilon>0$ and $u \in \tilde{P}$; note that $u=(\chi(\sigma), 0,0)$ for some unique $\sigma \in \omega^{\omega}$. Since $\alpha \notin A$ there exists some $s \prec \sigma$ such that $\alpha \notin A_{s}$ and if $|s|$ is big enough then $\operatorname{diam}\left(X \cap N_{s}\right)<\varepsilon$.

Then for all $t \in \omega^{<\omega}$ such that $s \prec t^{*}, \alpha \notin A_{t}$ hence $\varphi_{t}(\alpha)$ is connected. It follows by induction that for all $p \geq|s|$ the set $\bigcup_{s \preceq t,|t| \leq p} \varphi_{t}(\alpha)$ is connected, hence $\Phi(\alpha) \cap N_{s}={\overline{\bigcup_{s \preceq t} \varphi_{t}(\alpha)}}^{X}$ is connected.

Lemma 6.10. If $\alpha \in A$ then $\Phi(\alpha) \notin \mathcal{C}_{\text {loc }}(X)$.

Proof. Fix $\sigma \in \omega^{\omega}$ such that $\alpha \in A_{s}$ for all $s \prec \sigma$. We shall prove that the element $u=(\chi(\sigma), 0,0) \in \tilde{P}$ has no connected neighbourhood in $\Phi(\alpha)$.

So let $V$ be any neighbourhood of $u$ in $\Phi(\alpha)$. We can find $t \prec \sigma$ such that $N_{t} \subset V$ hence $\varphi_{t}(\alpha) \subset V$. Since $\alpha \in A_{t}$ there exists a clopen partition $\left(W, W^{*}\right)$ in $\varphi_{t}(\alpha)$ such that $a_{t} \in W$ and $a_{t}^{*} \in W^{*}$. Then setting $U=W \cup \overline{\bigcup_{s \succ t} \varphi_{s}(\alpha)}$ and $U^{*}=W^{*} \cup \overline{\bigcup_{s \nsucceq t} \varphi_{s}(\alpha)}$ we have $U \cap U^{*} \cap \tilde{P}=\emptyset$ hence $\left(U, U^{*}\right)$ is a partition of $\Phi(\alpha)$ into two relatively closed subsets, with $a_{t} \in U \cap V$ and $a_{t}^{*} \in U^{*} \cap V$, which proves that $V$ is not connected. 
To finish the proof of Theorem 6.4 observe that for any open set $V, \Phi^{-1}\left(V^{+}\right)=$ $\bigcup_{s \in \omega<\omega} \varphi_{s}^{-1}\left(V^{+}\right)$and since each mapping $\varphi_{s}$ is Borel then the mapping $\Phi$ is Borel too. Also by Lemma 6.9 and Lemma $6.10 \Phi$ reduces the set $2^{\omega} \backslash A$ to the set $\mathcal{C}_{\text {loc }}(X)$. Hence by Theorem 2.1 the set $\mathcal{C}_{\text {loc }}(X)$ is $\check{\mathcal{A}}\left(\mathbf{\Pi}_{1}^{1}\right)$-complete.

\section{Connectedness of open subsets}

Our goal in this section is to study the complexity of the set of all open connected subsets of a given space $X$ that we identify to the set

$$
\check{\mathcal{C}}(X)=\{F \in \mathcal{F}(X): X \backslash F \text { is connected }\} .
$$

Proposition 7.1. For any Polish space $X$ there exists a Borel mapping $\delta$ : $\mathcal{F}(X) \rightarrow \mathcal{F}(X \times \mathbb{R})$ which reduces the set $\check{\mathcal{C}}(X)$ to the set $\mathcal{C}(X \times \mathbb{R})$.

Proof. Set $J=] 0,+\infty[\approx \mathbb{R}$ and fix a compatible distance $d<1$ on $X$. For all $F \in \mathcal{F}^{*}(X)$ let $\delta(F)$ be the graph of the restriction of the function $d(\cdot, F)$ to $U=X \backslash F$, so:

$$
\delta(F)=\{(x, t) \in X \times J: d(x, F)=t\}
$$

and set $\delta(\emptyset)=X \times\{1\}$. Then for all $F \in \mathcal{F}(X), \delta(F)$ is a closed subset of $X \times J$, which is homeomorphic to $U$, hence the mapping $\delta: \mathcal{F}(X) \rightarrow \mathcal{F}^{*}(X \times J)$ reduces the set $\check{\mathcal{C}}(X)$ to the $\operatorname{set} \mathcal{C}(X \times J)$.

To see that $\delta$ is Borel, fix a countable basis $\left(U_{i}\right)_{i \in \omega}$ of open sets in $X$. Since $X$ is Polish we can also fix a sequence $\left(c_{k}\right)_{k \in \omega}$ of Borel functions $c_{k}: \mathcal{F}^{*}(X) \rightarrow X$ such that for all $F \in \mathcal{F}^{*}(X), c_{k}(F) \in F$ and the set $\left\{c_{k}(F): k \in \omega\right\}$ is dense in $F$ (see Section 3). In particular if $a_{j}=c_{j}(X)$ then $\left\{a_{j}: j \in \omega\right\}$ is dense in $X$. Then for any basic open set $V \times] s, t[$ in $X \times J$ and $F \neq \emptyset, \delta(F) \cap(V \times] s, t[) \neq \emptyset$ if and only if:

$$
\exists(n, i, j): a_{j} \in U_{i} \subset V, U_{i} \cap F=\emptyset \text { and }\left\{\begin{array}{l}
\forall k, d\left(a_{j}, c_{k}(F)\right) \geq s+2^{-n} \\
\exists k, d\left(a_{j}, c_{k}(F)\right)<t
\end{array}\right.
$$

which is a Borel condition on $F$.

Let $X$ be a Polish space and $\Gamma$ be any projective class. Then by Proposition 7.1 if $\mathcal{C}(X \times \mathbb{R})$ is in $\Gamma$ then $\breve{\mathcal{C}}(X)$ is in $\Gamma$, and if $\breve{\mathcal{C}}(X)$ is $\Gamma$-hard then $\mathcal{C}(X \times \mathbb{R})$ is $\Gamma$-hard. Note however that the converse is false: if $X=\mathbb{R}^{\omega} \approx X \times \mathbb{R}$ then since any Polish space can be embedded as a closed subset in $\mathbb{R}^{\omega}$, it follows from Theorem 4.2 that the set $\mathcal{C}\left(\mathbb{R}^{\omega}\right)$ is $\Pi_{2}^{1}$-complete while as we shall see in Proposition 7.5 the set $\check{\mathcal{C}}\left(\mathbb{R}^{\omega}\right)$ is Borel.

We recall that if the space $X$ is compact then $\mathcal{C}(X)$ is a compact subset of $\mathcal{K}(X)$. 
Proposition 7.2. If $X$ is a compact space then $\check{\mathcal{C}}(X)$ is a $\Pi_{1}^{1}$ subset of $\mathcal{K}(X)$.

Proof. As in the proof of Proposition 4.1 for any $F \in \mathcal{F}(X)$ we have $F \notin \check{\mathcal{C}}(X)$ if and only if:

$$
\exists F_{0}, F_{1} \in \mathcal{F}(X): F_{0} \cup F \neq X, F_{1} \cup F \neq X, F_{0} \cup F_{1}=X \text { and } F \supset F_{0} \cap F_{1}
$$

and since $X$ is compact then the relation " $F_{0} \cup F_{1}=X$ " is compact and the relation " $F^{\prime} \neq F$ " is open hence $\sigma$-compact, while the relation " $F \supset F_{0} \cap F_{1}$ " is $\mathbf{G}_{\delta}$ and it follows that the right hand side defines a $\boldsymbol{\Sigma}_{1}^{1}$ set hence $\check{\mathcal{C}}(X)$ is $\boldsymbol{\Pi}_{1}^{1}$.

Theorem 7.3. There exists a compact (connected) space $X \subset \mathbb{I}^{2}$ for which the set $\check{\mathcal{C}}(X)$ is $\boldsymbol{\Pi}_{1}^{1}$-complete.

Proof. Let $\mathbb{Q}$ be the set of all rational numbers in $\mathbb{I}$ and $\mathbb{P}=\mathbb{I} \backslash \mathbb{Q}$. Fix an enumeration $\left(q_{n}\right)_{n \geq 1}$ of $\mathbb{Q}$ and consider the Kuratowski-Sierpinski function $f$ : $\mathbb{P} \rightarrow \mathbb{I}$ (see [7]) defined by

$$
f(x)=\sum_{n=1}^{\infty} 2^{-n} \sin \left(\frac{1}{x-q_{n}}\right) .
$$

It is clear that the function $f$ is continuous on $\mathbb{P}$ but has no continuous extension to $\mathbb{I}$. More precisely for all $q \in \mathbb{Q}$ the set of all cluster values of $f$ at $q$ is a non trivial interval $J_{q}$. Note that the graph $G:=\operatorname{Gr}(f) \approx \mathbb{P}$ is not connected. However as shown in [7] (see also [2]) if $\tilde{f}: \mathbb{I} \rightarrow \mathbb{I}$ is any extension of $f$ with $\tilde{f}(q) \in J_{q}$ for all $q \in \mathbb{Q}$ then $\operatorname{Gr}(\tilde{f})$ is connected. In particular the closure of $G$ in $\mathbb{I}^{2}$ :

$$
X:=\bar{G}=G \cup \bigcup_{q \in \mathbb{Q}} J_{q}
$$

is a compact connected space. It follows that if $G \subset H \subset \bar{G}$ and for all $q \in \mathbb{Q}$, $H(q) \neq \emptyset$ then $H$ is connected.

We also need the following result from [2]. (Observe that the set $\bar{F}$ of Lemma 9.5 in [2] is the graph of a function $g$ with the properties stated in Lemma 7.4.)

Lemma 7.4. There exists a perfect zero-dimensional compact set $\{0,1\} \subset \Delta \subset \mathbb{I}$ such that $Q=\Delta \cap \mathbb{Q}$ is dense in $\Delta$ and $f_{\mid \Delta \cap \mathbb{P}}$ admits a continuous extension $g: \Delta \rightarrow \mathbb{I}$.

So let $(Q, \Delta)$ as in Lemma 7.4 and consider the mapping $\Phi: \mathcal{K}^{*}(\Delta) \rightarrow \mathcal{K}(X)$ defined by

$$
\Phi(K)=\operatorname{Gr}\left(g_{\mid K}\right) .
$$

Then $\Phi$ is clearly continuous and we now check that $\Phi$ reduces the $\Pi_{1}^{1}$-complete set $\mathbb{P}_{1}=\mathbb{P}_{1}(Q, \Delta)$ to $\check{\mathcal{C}}(X)$. So let $K \in \mathcal{K}^{*}(\Delta)$ and set $U=X \backslash \Phi(K)$ : 
- If $K \in \mathbb{P}_{1}$ then $K \subset \mathbb{Q}$; then for all $q \in \mathbb{Q}, U(q)=J_{q} \backslash\{g(q)\}$ if $q \in K$, and $U(q)=J_{q}$ if not. Hence for all $q \in \mathbb{Q}, U(q) \neq \emptyset$ so $U$ is connected, that is $\Phi(K) \in \check{\mathcal{C}}(X)$.

- If $K \notin \mathbb{P}_{1}$ then $K$ contains some irrational number $\alpha$ and then $\bar{G}(\alpha)=$ $\{f(\alpha)\}=\{g(\alpha)\}$ hence $U(\alpha)=\emptyset$ and we can write $U=(U \cap[0, \alpha[\times \mathbb{J}) \cup$ $(U \cap] \alpha, 1] \times \mathbb{J})$ which proves that $U$ is not connected, that is $\Phi(K) \notin \check{\mathcal{C}}(X)$.

We recall (Proposition 4.1) that if the space $X$ is Polish then $\mathcal{C}(X)$ is a $\Pi_{2}^{1}$ subset of $\mathcal{F}(X)$.

Proposition 7.5. a) If $X$ is a Polish space then $\check{\mathcal{C}}(X)$ is a $\Pi_{2}^{1}$ subset of $\mathcal{F}(X)$. b) If moreover $X$ is locally connected then $\check{\mathcal{C}}(X)$ is a $\boldsymbol{\Delta}_{1}^{1}$ subset of $\mathcal{F}(X)$, more precisely a $\Pi_{2}^{0}$ subset for any admissible topology on $\mathcal{F}(X)$.

Proof. a) Again for $F \in \mathcal{F}(X)$ we have $F \notin \check{\mathcal{C}}(X)$ if and only if:

$$
\exists F_{0}, F_{1} \in \mathcal{F}(X): F_{0} \cup F \neq X, F_{1} \cup F \neq X, F_{0} \cup F_{1}=X \text { and } F \supset F_{0} \cap F_{1}
$$

and by the same arguments as in the proof of Proposition 4.1 one can check that the right hand side defines a $\boldsymbol{\Sigma}_{2}^{1}$ set hence $\check{\mathcal{C}}(X)$ is a $\boldsymbol{\Pi}_{2}^{1}$ set.

b) Suppose that $X$ is locally connected. Fix a countable basis $\left(U_{i}\right)_{i \in \omega}$ of open sets in $X$. Let $U$ be any open subset of $X$, since $U$ is also a Polish locally connected space, then $U$ is connected if and only if it is pathwise connected. Hence for any $F \in \mathcal{F}(X), F \in \check{\mathcal{C}}(X)$ if and only if:

$$
(\star)\left\{\begin{array}{l}
\forall i, j, F \cap U_{i} \neq \emptyset \text { or } F \cap U_{j} \neq \emptyset \text { or } \exists K \subset X \text { such that: } K \cap F=\emptyset, \\
K \cap U_{i} \neq \emptyset, K \cap U_{j} \neq \emptyset, \text { and } K \text { is compact and connected }
\end{array}\right.
$$

Note that if $U \subset X$ is open and $K \subset X$ is compact then for any admissible topology on $\mathcal{F}(X)$ the sets

$$
U^{+}=\{F \in \mathcal{F}(X): F \cap U \neq \emptyset\} \text { and } K^{-}=\{F \in \mathcal{F}(X): F \cap K=\emptyset\}
$$

are open subsets of $\mathcal{F}(X)$. Hence if $\mathcal{E}_{i, j}$ denotes the set of all compact subsets of $X$ satisfying the conditions in the second line of $(\star)$ then

$$
\check{\mathcal{C}}(X)=\bigcap_{i, j}\left(U_{i}^{+} \cup U_{j}^{+} \cup \bigcup_{K \in \mathcal{E}_{i, j}} K^{-}\right)
$$

Hence $\check{\mathcal{C}}(X)$ is a $\Pi_{2}^{0}$ subset of $\mathcal{F}(X)$.

Theorem 7.6. There exists a Polish space $X \subset \mathbb{I}^{2}$ for which the set $\check{\mathcal{C}}(X)$ is $\boldsymbol{\Pi}_{2}^{1}$-complete. 
For the proof we need the following result which can be viewed as a kind of dual version of Lemma 3.5 for $n=2$. We recall that $\mathcal{K}^{*}(X)$ denotes the space of all non empty compact subsets of $X$.

Lemma 7.7. Let $\Delta \approx 2^{\omega}$. Then for any $\boldsymbol{\Sigma}_{2}^{1}$ set $A \subset 2^{\omega}$ there exists a $\Pi_{2}^{0}$ set $G \subset \Delta \times \Delta$ and a continuous mapping $\Phi: \alpha \mapsto K_{\alpha}$ from $2^{\omega}$ to $\mathcal{K}^{*}(G)$ such that

$$
A=\left\{\alpha \in 2^{\omega}: \exists x \in \Delta, G(x)=K_{\alpha}(x)\right\}
$$

Proof. We may suppose that $\Delta=2^{\omega}$. Fix a homeomorphism $\imath:(\alpha, \beta) \mapsto\langle\alpha, \beta\rangle$ from $2^{\omega} \times 2^{\omega}$ onto $2^{\omega}$. Let $Q=\left\{\varepsilon \in 2^{\omega}: \exists n, \forall m \geq n, \varepsilon(m)=0\right\}$ and $P=$ $2^{\omega} \backslash Q \approx \omega^{\omega}$. We denote by $\mathbf{0}$ the element $\varepsilon$ of $Q$ such that for all $n, \varepsilon(n)=0$.

Let $A$ be any $\Sigma_{2}^{1}$ subset of $2^{\omega}$, fix a $\Pi_{1}^{1}$ set $B \subset 2^{\omega} \times 2^{\omega}$ such that $A$ is the projection of $B$ on the first factor. Since the set $2^{\omega} \backslash \imath(B)$ is $\Sigma_{1}^{1}$ it is the projection of some closed subset of $2^{\omega} \times P$, so we can fix a compact set $H$ of $2^{\omega} \times 2^{\omega}$ such that $2^{\omega} \backslash \imath(B)$ is the projection of $H \cap\left(2^{\omega} \times P\right)$. We then define

$$
G=\left(2^{\omega} \times\{\mathbf{0}\}\right) \cup\left(H \cap\left(2^{\omega} \times P\right)\right)
$$

and for all $\alpha \in 2^{\omega}$,

$$
K_{\alpha}=\imath\left(\{\alpha\} \times 2^{\omega}\right) \times\{\mathbf{0}\} \subset G .
$$

Then clearly $G$ is a $\Pi_{2}^{0}$ set, and the mapping $\Phi: \alpha \mapsto K_{\alpha}$ is an embedding of $2^{\omega}$ into $\mathcal{K}^{*}(G)$; and we now verify the conclusion of the lemma.

If $\alpha \in A$ we can find $\beta$ such that $(\alpha, \beta) \in B$ then setting $x=\imath(\alpha, \beta)$ we have $(x, \mathbf{0}) \in K_{\alpha}$ and since $x \in \imath(B)$ then there is no $\varepsilon \in P$ such that $(x, \varepsilon) \in H$ hence $G(x)=\{\mathbf{0}\} \subset K_{\alpha}(x)$.

Conversely if $\alpha \in 2^{\omega}$ and for some $x \in 2^{\omega}, G(x) \subset K_{\alpha}(x)=\{\mathbf{0}\} \subset G(x)$ then we necessarily have $G(x)=K_{\alpha}(x)=\{\mathbf{0}\}$. But by the definition of $K_{\alpha}$ we can write $x=\imath(\alpha, \beta)$ for some $\beta$ and we claim that $(\alpha, \beta) \in B$. For otherwise $x$ would be in $2^{\omega} \backslash \imath(B)$, and then there would exist some $\varepsilon \in P$, such that $(x, \varepsilon) \in H$ hence $\varepsilon \in G(x)=\{\mathbf{0}\}$ which is a contradiction since $\mathbf{0} \notin P$. This proves that $(\alpha, \beta) \in B$ hence $\alpha \in A$.

Proof of Theorem 7.6: Fix $\Delta \subset] 0,1\left[\right.$ with $\Delta \approx 2^{\omega}$ and $A \subset 2^{\omega}$ a complete $\boldsymbol{\Sigma}_{2}^{1}$ set. Let $G$ and $\Phi$, associated to $A$ as in Lemma 7.7 and consider the set

$$
X=((\mathbb{I} \backslash \Delta) \times \mathbb{I}) \cup G
$$

which is clearly a $\Pi_{2}^{0}$ subset of $\mathbb{I}^{2}$. Since for all $\alpha, K_{\alpha} \subset G$ we may view $\Phi$ as a continuous mapping from $2^{\omega}$ into $\mathcal{F}(X)$ with for all $\alpha$ :

$$
U_{\alpha}:=X \backslash \Phi(\alpha)=((\mathbb{I} \backslash \Delta) \times \mathbb{I}) \cup\left(G \backslash K_{\alpha}\right)
$$


and to finish the proof of Theorem 7.6 we now show that $U_{\alpha}$ is connected if and only if $\alpha \notin A$.

Lemma 7.8. If $\alpha \in A$ then $U_{\alpha}$ is not connected.

Proof. If $\alpha \in A$ then by definition there exists some $x^{*} \in \Delta$ such that $G\left(x^{*}\right)=$ $K_{\alpha}\left(x^{*}\right)$, so $U_{\alpha} \cap\left(\left\{x^{*}\right\} \times \mathbb{I}\right)=\emptyset$. Hence the sets $U_{0}=U \cap\left(\left[0, x^{*}[\times \mathbb{I})\right.\right.$ and $U_{1}=$ $\left.\left.U \cap(] x^{*}, 1\right] \times \mathbb{I}\right)$ form a clopen partition of $U$ with $(0,0) \in U_{0}$ and $(1,0) \in U_{1}$, which proves that $U$ is not connected.

Lemma 7.9. If $\alpha \notin A$ then $U_{\alpha}$ is connected.

Proof. The argument is quite similar to the one of Theorem 4.2.

Suppose by contradiction that $\left(V_{0}, V_{1}\right)$ is a clopen partition of $U_{\alpha}$ with $(0,0) \in$ $V_{0}$. Let $\mathcal{J}$ denote the set of all connected components of $\mathbb{I} \backslash \Delta$, and for $\varepsilon=0,1$ let $\mathcal{J}_{\varepsilon}=\left\{J \in \mathcal{J}: J \times \mathbb{I} \subset V_{\varepsilon}\right\}$. Note that for all $J \in \mathcal{J}$ we have $J \times \mathbb{I} \subset U_{\alpha} \subset V_{0} \cup V_{1}$, hence by connectedness either $J \in \mathcal{J}_{0}$ or $J \in \mathcal{J}_{1}$.

We claim that $\mathcal{J}=\mathcal{J}_{0}$. For otherwise, for any interval $J \subset \mathbb{I}$ set $x_{J}:=\min (J)$, and consider $x_{*}=\inf \left\{x_{J}: J \in \mathcal{J}_{1}\right\}$. Then clearly $x^{*} \in \Delta$ and since $\alpha \notin A$ we can find $y_{*} \in \mathbb{I}$ such that $\left(x_{*}, y_{*}\right) \in G \backslash K_{\alpha}$. Note that since $0 \notin \Delta$ then $x_{*}>0$ and for any $J \in \mathcal{J}$ if $x^{J}<x_{*}$ then $J \in \mathcal{J}_{0}$ hence $J \times \mathbb{I} \subset V_{0}$, and since $V_{0}$ is a closed subset of $U_{\alpha}$ then $\left(x_{*}, y_{*}\right) \in V_{0}$. Also from the definition of $x_{*}$ we can find a sequence $\left(J_{n}\right)$ in $\mathcal{J}_{1}$, such that $x_{*}=\lim _{n} x_{J_{n}}$. Then $J_{n} \times \mathbb{I} \subset V_{1}$, and again since $V_{1}$ is a closed subset of $U_{\alpha}$ then $\left(x_{*}, y_{*}\right) \in V_{1}$. Hence $\left(x_{*}, y_{*}\right) \in V_{0} \cap V_{1}$ which is a contradiction.

This finishes the proof of Theorem 7.6.

\section{References}

[1] J. Burgess, Classical hierarchies from a modern standpoint. I. C-sets, Fund. Math. 115 (1983), 81-95.

[2] G. Debs and J. Saint Raymond, The descriptive complexity of the set of all closed zero-dimensional subsets of a Polish space. Topology and its Appl. 248 (2018), 43-66.

[3] G. Debs and J. Saint Raymond, On כГ-complete sets, To appear in Proc. Amer. Math. Soc.

[4] A. Kechris, On the concept of $\boldsymbol{\Pi}_{1}^{1}$-completeness, Proc. Amer. Math. Soc. 125 (1997), 1811-1814. 
[5] A. Kechris, Classical descriptive set theory. Graduate Texts in Mathematics, 156. Springer-Verlag, New York, 1995.

[6] K. Kuratowski, Topology Vol. I. New edition, revised and augmented. Translated from the French by J. Jaworowski, Academic Press, New York-London; Państwowe Wydawnictwo Naukowe, Warsaw (1966).

[7] K. Kuratowski and W. Sierpinski, Les fonctions de classe 1 et les ensembles connexes punctiformes, Fund. Math. 3 (1922), 303-313.

[8] Y. N. Moschovakis, Descriptive Set Theory, Studies in logic 100 NorthHolland, Amsterdam (1979); second edition: Mathematical surveys and monographs 155, Amer. Math. Soc. Providence, Rhode Island (2009). 